\title{
Responses of a two degree-of-freedom system coupled to a nonlinear damper under multi-forcing frequencies
}

\author{
Sergio Bellizzi ${ }^{\mathrm{a}, *}$, Renaud Côte ${ }^{\mathrm{b}}$, Marc Pachebat ${ }^{\mathrm{a}}$, \\ ${ }^{a} L M A, C N R S$, UPR 7051, Centrale Marseille, Aix-Marseille Univ \\ F-13420 Marseille Cedex 20, France. \\ ${ }^{b}$ LMA, Aix-Marseille Univ, CNRS, UPR 7051, Centrale Marseille \\ F-13451 Marseille, France.
}

\begin{abstract}
In this paper, forced responses are investigated in a two degree-of-freedom linear system with a linear coupling to a Nonlinear Energy Sink (NES) subjected to quasi-periodic excitation. The quasi-periodic regimes associated to quasi-periodic forcing in the regime of 1:1-1:1 are studied analytically using the complexification method combined to the averaging method in terms of multi-time parameter. Local bifurcations of the quasi-periodic regimes are also analyzed using the excitation frequencies as control parameters. The nonlinear differential system is also solved numerically in time domain and the responses are analyzed in view of the analytical results. Stable and unstable quasi-periodic responses are found in good agreement with the analytical study, and strongly modulated responses are noticed. We observe that a single NES can be efficient for the reduction of two resonance peaks even if they are well separated, incommensurable, and excited simultaneously.
\end{abstract}

Keywords: Nonlinear absorber, Nonlinear energy sink, Targeted energy transfer, Quasiperiodic forcing, Complexification method, Stability

\footnotetext{
${ }^{*}$ Corresponding Author

Email address: bellizzi@lma.cnrs-mrs.fr (Sergio Bellizzi)
} 


\section{Nomenclature}

$\eta \quad$ viscous damping coefficient of membrane

$\nu \quad$ Poisson ratio of membrane

$\omega_{i}^{\mathrm{p}} \quad$ first resonance frequency of the tube $i$

$\rho_{0} \quad$ Air density

$\rho_{\mathrm{m}} \quad$ Membrane density

$\tau_{i} \quad$ damping ratio of the 1-DOF system modeling the tube $i$

$c_{0} \quad$ sound wave velocity

$d_{i} \quad$ diameter of pipe $i$

$d_{\mathrm{m}} \quad$ diameter of membrane

E Young's modulus of membrane

$h_{\mathrm{m}} \quad$ thickness of membrane

$k_{3} \quad$ cubic stiffness of the 1-DOF system modeling of the NES

$k_{i} \quad$ stiffness of the 1-DOF system modeling the tube $i$

$k_{\mathrm{m}} \quad$ linear stiffness of the 1-DOF system modeling of the NES

$L_{i} \quad$ length of pipe $i$

$m_{i} \quad$ mass of the 1-DOF system modeling the tube $i$

$m_{\mathrm{m}}$ mass of the 1-DOF system modeling of the NES

$R_{\mathrm{m}} \quad$ radius of membrane

$S_{i} \quad$ area of pipe $i$

$S_{\mathrm{m}} \quad$ area of membrane

$V_{\mathrm{m}} \quad$ volume of the coupling box: pipes/NES 


\section{Introduction}

A series of papers $[1,2,3,4]$ demonstrated that a passive control of sound at low frequencies can be achieved using a vibroacoustic coupling between the acoustic field (the primary system) and a geometrically nonlinear thin baffled structure (the nonlinear absorber). In [1,2], the thin baffled structure consists of a simple thin circular visco-elastic membrane whereas in $[3,4]$ a loudspeaker used as a suspended piston is considered. In the four papers, theoretical and experimental results are reported considering transient and periodic external excitation. The reduction principle of sound is based on the phenomenon called Targeted Energy Transfer (TET) or Energy Pumping [5]. If the nonlinear absorber is properly designed for the primary system, an irreversible energy transfer from the linear system toward the absorber occurs, the energy is dissipated within the absorber damper and the forced dynamic response of the primary system is limited [6]. This means that the nonlinear system behaves like a "sink" where there is motion localization and energy dissipation. In literature, this is also called Nonlinear Energy Sink (NES). The complex dynamics of this kind of coupled systems can be described in terms of resonance capture or nonlinear normal modes [5].

Under periodic external excitation applied to the primary system, the nonlinear absorbers can efficiently reduce the resonance peak by entering the whole system in a quasi-periodic motion with repetitive TET phase [6]. Weakly quasi-periodic responses and strongly quasi-periodic responses (also named "strongly modulated responses") can exist or coexist $[7,8]$. The very important point is that this peak reduction can occur in a wide frequency band, with the NES adapting itself to the resonance frequencies of the primary system. On the other hand, the NES can operate efficiently only in a limited range of the amplitude of the primary system. Following these results, design and optimization of the nonlinear absorber have been addressed in $[9,10]$.

Similar tools have been used to investigate a two Degree-Of-Freedom (DOF) linear systems with only one attached NES. An analysis of a competitive energy transfer between a two DOF linear system and the NES in terms of transient dynamic was presented in [11] exhibiting two activation energy thresholds and proposing scenarios to forecast the TET mechanics. Periodic external excitation was considered in [12] where the ability of the NES to reduce the vibration from both excited modes of the primary system is demonstrated. 
In the works exposed above, TET was shown for single frequency, two frequency or broadband excitation spectrum [13], but always close to one single resonance frequency of the primary (linear) system. In these situations a NES is a more effective vibration absorber than usual linear dampers, mainly because its action is not limited to the immediate vicinity of a single fixed frequency. The question that arises is the width of the efficient range of a NES: can a single NES reduce well-separated resonance peaks of a primary system that are excited at the same time? The present work looks for the existence of this property that could broaden the applications of TET.

In this study, an acoustic medium (as in $[1,2,3,4]$ ) is considered as a primary system coupled to a simple thin circular visco-elastic membrane (as in $[1,2]$ ). However, two significant differences can be highlighted from these past studies: firstly, the acoustic medium is modeled using two modes and secondly the coupling effect between the primary system and the NES is analyzed under two different harmonic excitations.

The paper is organized as follows. In Section 2, the system under study is described and modeled as a two DOF linear acoustic system coupled to a NES. We establish the equations of motion that will be transformed along the analytic treatment. In Section 3, we express the system response to periodic and quasiperiodic excitation using a complexification method, and we express the stability analysis of these solutions. Complexification is used to split solutions into fast and slow components. Slow components can be seen as a slowly varying amplitude of an oscillation. These different time scales permit to separate the variables: the fast components are assumed to be at the source frequencies, the slow are found by averaging the equations in terms of multi-time parameters. In this process, several terms are neglected. In order to control the magnitude of these terms, the equations are written in dimensionless form prior to complexification. This process ends up with an autonomous set of differential equations, and solutions appear in the form of polynomial roots. The stability and local bifurcation analysis are done by monitoring the evolution of small perturbations. In Section 4, we apply the general formulas established before on a realistic case. We establish the responses to periodic, then quasiperiodic excitations and point out the differences. Next we check the validity of these results with direct numerical integration of the dimensional equations of motion. In Section, 5 we gather the main observations and we conclude. 


\section{Description of the vibroacoustic system}

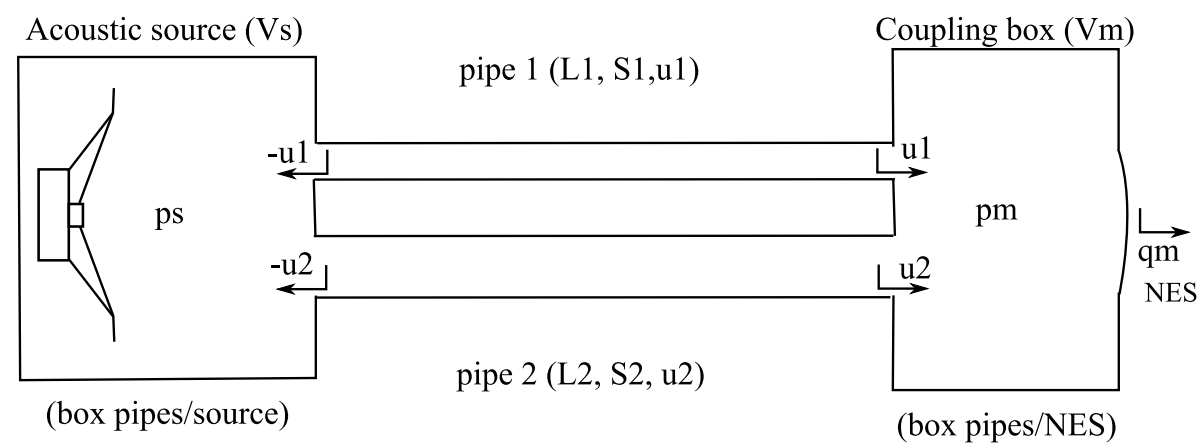

Figure 1: Schema of the vibroacoustic system.

The system under study is shown Fig. 1. It consists of an acoustic medium coupled to a simple thin circular clamped visco-elastic membrane by means of a coupling box. The acoustic medium is composed by two pipes of different lengths and section areas opened on both ends. In practical terms, the length can be adjusted using U-shaped pipes. The coupling between the pipes and the membrane is ensured acoustically by the air in a coupling box, which is sufficiently large to give a weak linear coupling stiffness. A pre-stress can be imposed at the membrane boundaries. An acoustic source consisting of a loudspeaker and a coupling box which is connected to the entrance of both pipes is used.

\subsection{Associated model}

Following [2] and [3] and under the same assumptions, a simple model to predict qualitatively the behavior of the vibroacoustic system can be obtained corresponding to the following equations of motion.

The equation of motion of the pipe 1 is given by

$$
m_{1} \ddot{u}_{1}(t)+2 \tau_{1} \sqrt{k_{1} m_{1}} \dot{u}_{1}(t)+k_{1} u_{1}(t)=-S_{1} p_{\mathrm{m}}(t)-S_{1} p_{\mathrm{s}}(t)
$$

where $u_{1}(t)$ denotes the acoustic displacement at the end of the pipe, $p_{\mathrm{m}}(t)$ denotes the pressure in the coupling box pipes/NES, $p_{\mathrm{s}}(t)$ denotes the pressure in the coupling box pipes/source and the parameters satisfy

$$
m_{1}=\frac{\rho_{0} S_{1} L_{1}}{2} \text { and } k_{1}=\frac{\rho_{0} c_{0}^{2} \pi^{2} S_{1}}{2 L_{1}} \text { giving } \omega_{1}^{\mathrm{p}^{2}}=\frac{k_{1}}{m_{1}}=\frac{c_{0}^{2} \pi^{2}}{L_{1}^{2}} .
$$


The equation of motion of the pipe 2 is given by

$$
m_{2} \ddot{u}_{2}(t)+2 \tau_{2} \sqrt{k_{2} m_{2}} \dot{u}_{2}(t)+k_{2} u_{2}(t)=-S_{2} p_{\mathrm{m}}(t)-S_{2} p_{\mathrm{s}}(t)
$$

where $u_{2}(t)$ denotes the acoustic displacement at the end of the pipe and the parameters satisfy

$$
m_{2}=\frac{\rho_{0} S_{2} L_{2}}{2} \text { and } k_{2}=\frac{\rho_{0} c_{0}^{2} \pi^{2} S_{2}}{2 L_{2}} \text { giving } \omega_{2}^{\mathrm{p}^{2}}=\frac{c_{0}^{2} \pi^{2}}{L_{2}^{2}} .
$$

The equation of motion of the pre-stressed membrane (NES) is given by

$m_{\mathrm{m}} \ddot{q}_{\mathrm{m}}(t)+k_{\mathrm{m}}\left(\frac{f_{1}^{2}}{f_{0}^{2}} q_{\mathrm{m}}(t)+\eta \dot{q}_{\mathrm{m}}(t)\right)+k_{3}\left(q_{\mathrm{m}}^{3}(t)+2 \eta\left|q_{\mathrm{m}}(t)\right|^{2} \dot{q}_{\mathrm{m}}(t)\right)=\frac{S_{\mathrm{m}}}{2} p_{\mathrm{m}}(t)$

where $q_{\mathrm{m}}(t)$ denotes the transversal displacement of the center of the membrane and the parameters satisfy

$$
\begin{gathered}
m_{\mathrm{m}}=\frac{\rho_{\mathrm{m}} S_{\mathrm{m}} h_{\mathrm{m}}}{3}, k_{\mathrm{m}}=\frac{1.015^{4} \pi^{5}}{36} \frac{E h_{\mathrm{m}}^{3}}{\left(1-\nu^{2}\right) R_{\mathrm{m}}^{2}}, \\
f_{0}=\frac{1}{2 \pi} \sqrt{\frac{1.015^{4} \pi^{4} E h_{\mathrm{m}}^{2}}{12\left(1-\nu^{2}\right) \rho_{\mathrm{m}} R_{m}^{4}}} \text { and } k_{3}=\frac{8 \pi E h_{\mathrm{m}}}{3\left(1-\nu^{2}\right) R_{\mathrm{m}}^{2}} .
\end{gathered}
$$

Here $f_{0}$ denotes the first resonance frequency of the membrane without prestress and $f_{1}$ denotes the first resonance frequency of the membrane with prestress. The resonance frequency $f_{1}$ can be measured experimentally so it can be considered as a parameter of the model. As described in [2], the nonlinear equation of motion (5) has been obtained considering the membrane as a thin elastic structure with geometric nonlinearities and using a one DOF Rayleigh-Ritz reduction with a single parabolic shape function to describe the transversal displacement of the membrane. All details can be found in $[2]$.

The vibroacoustic coupling between the two pipes and the membrane is given by the acoustic pressure $p_{m}(t)$ into the coupling box pipes/NES, which is dependent on $u_{1}(t), u_{2}(t)$ and $q_{\mathrm{m}}(t)$ accordingly to

$$
p_{\mathrm{m}}(t)=k_{b}\left(-\frac{S_{\mathrm{m}}}{2} q_{\mathrm{m}}(t)+S_{1} u_{1}(t)+S_{2} u_{2}(t)\right) \text { with } k_{b}=\frac{\rho_{0} c_{0}^{2}}{V_{\mathrm{m}}} .
$$


For simplicity, the coupling box pipes/source and the loudspeaker are not modeled. We assume that the acoustic source is characterized by the acoustic pressure $p_{\mathrm{s}}(t)$ into the coupling box pipes/source. In case of bi-periodic (or quasi-periodic) excitation, the acoustic pressure is of the form

$$
p_{\mathrm{s}}(t)=E_{1} \cos \left(\omega_{1}^{\mathrm{s}} t+\varphi_{1}^{\mathrm{s}}\right)+E_{2} \cos \left(\omega_{2}^{\mathrm{s}} t+\varphi_{2}^{\mathrm{s}}\right)
$$

where $\omega_{1}^{\mathrm{s}}$ and $\omega_{2}^{\mathrm{s}}$ are two incommensurable frequencies and $\varphi_{1}^{\mathrm{s}}$ and $\varphi_{2}^{\mathrm{s}}$ are two arbitrary phases.

Hence the dimensional equations of motion read as

$$
\begin{aligned}
& m_{1} \ddot{u}_{1}(t)+2 \tau_{1} \sqrt{k_{1} m_{1}} \dot{u}_{1}(t)+k_{1} u_{1}(t) \\
& \quad+S_{1} k_{b}\left(S_{1} u_{1}(t)+S_{2} u_{2}(t)-\frac{S_{\mathrm{m}}}{2} q_{\mathrm{m}}(t)\right)=-S_{1} p_{s}(t), \\
& m_{2} \ddot{u}_{2}(t)+2 \tau_{2} \sqrt{k_{2} m_{2}} \dot{u}_{2}(t)+k_{2} u_{2}(t) \\
& \quad+S_{2} k_{b}\left(S_{1} u_{1}(t)+S_{2} u_{2}(t)-\frac{S_{\mathrm{m}}}{2} q_{\mathrm{m}}(t)\right)=-S_{2} p_{s}(t), \\
& m_{\mathrm{m}} \ddot{q}_{\mathrm{m}}(t)+f\left(\dot{q}_{\mathrm{m}}(t), q_{\mathrm{m}}(t)\right) \\
& \quad-\frac{S_{\mathrm{m}}}{2} k_{b}\left(S_{1} u_{1}(t)+S_{2} u_{2}(t)-\frac{S_{\mathrm{m}}}{2} q_{\mathrm{m}}(t)\right)=0
\end{aligned}
$$

where

$$
f(\dot{x}, x)=k_{\mathrm{m}}\left(\frac{f_{1}^{2}}{f_{0}^{2}} x+\eta \dot{x}\right)+k_{3}\left(x^{3}+2 \eta|x|^{2} \dot{x}\right)
$$

\subsection{Nondimensional equations of motion}

Eqs. (10-11) are first rewritten in the matrix form

$$
\mathbf{M} \ddot{\mathbf{U}}(t)+\mathbf{C} \dot{\mathbf{U}}(t)+\mathbf{K U}(t)-k_{b} \frac{S_{\mathrm{m}}}{2}\left(\begin{array}{c}
S_{1} \\
S_{2}
\end{array}\right) q_{\mathrm{m}}(t)=-\left(\begin{array}{c}
S_{1} \\
S_{2}
\end{array}\right) p_{s}(t)
$$

where

$$
\mathbf{U}(t)=\left(\begin{array}{l}
u_{1}(t) \\
u_{2}(t)
\end{array}\right)
$$

and the matrices $\mathbf{M}, \mathbf{C}$ and $\mathbf{K}$ are easily deduced from Eqs. (10-11).

Introducing the following change of variable

$$
\mathbf{U}(t)=\boldsymbol{\Phi} \mathbf{V}(t) \text { with } \mathbf{V}(t)=\left(\begin{array}{l}
v_{1}(t) \\
v_{2}(t)
\end{array}\right)
$$


where the modal matrix

$$
\boldsymbol{\Phi}=\left[\boldsymbol{\Phi}_{1} \boldsymbol{\Phi}_{2}\right]=\left(\begin{array}{ll}
\Phi_{11} & \Phi_{12} \\
\Phi_{21} & \Phi_{22}
\end{array}\right)
$$

is defined following the relations

$$
\mathbf{K} \boldsymbol{\Phi}_{i}=\omega_{i}^{\mathrm{a}^{2}} \mathbf{M} \boldsymbol{\Phi}_{i} \text { for } i=1,2 \text { with } \omega_{1}^{\mathrm{a}} \leq \omega_{2}^{\mathrm{a}} \text { and } \boldsymbol{\Phi}^{T} \mathbf{M} \boldsymbol{\Phi}=\mathbf{M},
$$

Eqs. (10-12) read as

$$
\begin{aligned}
& m_{1} \ddot{v}_{1}(t)+\bar{c}_{11} \dot{v}_{1}(t)+\bar{c}_{12} \dot{v}_{2}(t)+m_{1} \omega_{1}^{\mathrm{a}^{2}} v_{1}(t) \\
& \quad-\frac{S_{\mathrm{m}}}{2} k_{b} \bar{S}_{1} q_{\mathrm{m}}(t)=-\bar{S}_{1} p_{s}(t), \\
& m_{2} \ddot{v}_{2}(t)+\bar{c}_{21} \dot{v}_{1}(t)+\bar{c}_{22} \dot{v}_{2}(t)+m_{2} \omega_{2}^{\mathrm{a}^{2}} v_{2}(t) \\
& \quad-\frac{S_{\mathrm{m}}}{2} k_{b} \bar{S}_{2} q_{\mathrm{m}}(t)=-\bar{S}_{2} p_{s}(t), \\
& m_{\mathrm{m}} \ddot{q}_{\mathrm{m}}(t)+f\left(\dot{q}_{\mathrm{m}}(t), q_{\mathrm{m}}(t)\right) \\
& \quad-\frac{S_{\mathrm{m}}}{2} k_{b}\left(\bar{S}_{1} v_{1}(t)+\bar{S}_{2} v_{2}(t)-\frac{S_{\mathrm{m}}}{2} q_{\mathrm{m}}(t)\right)=0
\end{aligned}
$$

where

$$
\boldsymbol{\Phi}^{T} \mathbf{C} \boldsymbol{\Phi}=\left(\begin{array}{cc}
\bar{c}_{11} & \bar{c}_{12} \\
\bar{c}_{21} & \bar{c}_{22}
\end{array}\right) \text { and } \boldsymbol{\Phi}^{T}\left(\begin{array}{c}
S_{1} \\
S_{2}
\end{array}\right)=\left(\begin{array}{c}
\bar{S}_{1} \\
\bar{S}_{2}
\end{array}\right) .
$$

Using now the following rescaled quantities

$$
x_{1}(t)=\frac{2 \bar{S}_{1}}{S_{\mathrm{m}}} \frac{v_{1}(t)}{h_{\mathrm{m}}}, x_{2}(t)=\frac{2 \bar{S}_{2}}{S_{\mathrm{m}}} \frac{v_{2}(t)}{h_{\mathrm{m}}} \text { and } x_{3}(t)=\frac{q_{\mathrm{m}}(t)}{h_{\mathrm{m}}},
$$

and the time normalization

$$
\tilde{x}_{1}(\tau)=x_{1}\left(\frac{\tau}{\omega_{1}^{\mathrm{p}}}\right), \tilde{x}_{2}(\tau)=x_{2}\left(\frac{\tau}{\omega_{1}^{\mathrm{p}}}\right), \tilde{x}_{3}(\tau)=q\left(\frac{\tau}{\omega_{1}^{\mathrm{p}}}\right) \text { with } \tau=\omega_{1}^{\mathrm{p}} t
$$

Eqs (18-20) take the following nondimensional form

$$
\begin{aligned}
\ddot{\tilde{x}}_{1}(\tau)+\lambda_{11} \dot{\tilde{x}}_{1}(\tau)+\lambda_{12} \dot{\tilde{x}}_{2}(\tau)+\omega_{1}^{2} \tilde{x}_{1}(\tau)-\beta_{1} \tilde{x}_{3}(\tau) & =-F_{1} \tilde{p}_{\mathrm{s}}(\tau), \\
\ddot{\tilde{x}}_{2}(\tau)+\lambda_{21} \dot{\tilde{x}}_{1}(\tau)+\lambda_{22} \dot{\tilde{x}}_{2}(\tau)+\omega_{2}^{2} \tilde{x}_{2}(\tau)-\beta_{2} \tilde{x}_{3}(\tau) & =-F_{2} \tilde{p}_{\mathrm{s}}(\tau), \\
\gamma \ddot{\tilde{x}}_{3}(\tau)+C_{1} \dot{\tilde{x}}_{3}(\tau)+C_{3} \tilde{x}_{3}(\tau)^{2} \dot{\tilde{x}}_{3}(\tau)+K_{1} \tilde{x}_{3}(\tau) & \\
+K_{3} \tilde{x}_{3}(\tau)^{3}+\beta_{1}\left(-\tilde{x}_{1}(\tau)-\tilde{x}_{2}(\tau)+\tilde{x}_{3}(\tau)\right) & =0
\end{aligned}
$$


where

$$
\begin{gathered}
\omega_{1}=\frac{\omega_{1}^{\mathrm{a}}}{\omega_{1}^{\mathrm{p}}}, \omega_{2}=\frac{\omega_{2}^{\mathrm{a}}}{\omega_{1}^{\mathrm{p}}} \\
\lambda_{11}=2\left(\tau_{1} \Phi_{11}^{2}+\tau_{2} \frac{S_{2}}{S_{1}} \Phi_{21}^{2}\right), \lambda_{12}=2\left(\tau_{1} \Phi_{11} \Phi_{12}+\tau_{2} \frac{S_{2}}{S_{1}} \Phi_{21} \Phi_{22}\right) \frac{\bar{S}_{1}}{\bar{S}_{2}} \\
\beta_{1}=\frac{2 S_{1} L_{1}}{\pi^{2} V_{\mathrm{m}}}\left(\Phi_{11}+\Phi_{21} \frac{S_{2}}{S_{1}}\right)^{2}, F_{1}=\frac{4 S_{1} L_{1}}{\rho_{0} c_{0}^{2} \pi^{2} S_{\mathrm{m}} h_{\mathrm{m}}}\left(\Phi_{11}+\Phi_{21} \frac{S_{2}}{S_{1}}\right)^{2} \\
\lambda_{21}=2\left(\tau_{1} \frac{S_{1}}{S_{2}} \Phi_{11} \Phi_{12}+\tau_{2} \Phi_{21} \Phi_{22}\right) \frac{\bar{S}_{2}}{\bar{S}_{1}} \frac{L_{1}}{L_{2}}, \lambda_{22}=2\left(\tau_{1} \frac{S_{1}}{S_{2}} \Phi_{12}^{2}+\tau_{2} \Phi_{22}^{2}\right) \frac{L_{1}}{L_{2}} \\
\beta_{2}=\frac{2 S_{2} L_{2}}{\pi^{2} V_{\mathrm{m}}}\left(\Phi_{12} \frac{S_{1}}{S_{2}}+\Phi_{22}\right)^{2} \frac{L_{1}^{2}}{L_{2}^{2}}, F_{2}=\frac{4 S_{2} L_{2}}{\rho_{0} c_{0}^{2} \pi^{2} S_{\mathrm{m}} h_{\mathrm{m}}}\left(\Phi_{12} \frac{S_{1}}{S_{2}}+\Phi_{22}\right)^{2} \frac{L_{1}^{2}}{L_{2}^{2}} \\
\gamma=\frac{8}{3} \frac{\rho_{\mathrm{m}}}{\rho_{0}} \frac{h_{\mathrm{m}} S_{1}}{S_{\mathrm{m}} L_{1}}\left(\Phi_{11}+\Phi_{21} \frac{S_{2}}{S_{1}}\right)^{2}, C_{1}=\frac{8 S_{1}}{\pi \rho_{0} c_{0} S_{\mathrm{m}}^{2}} k_{\mathrm{m}} \eta\left(\Phi_{11}+\Phi_{21} \frac{S_{2}}{S_{1}}\right)^{2} \\
C_{3}=\frac{16 S_{1} h_{\mathrm{m}}^{2}}{\rho_{0} c_{0} \pi S_{\mathrm{m}}^{2}} k_{3} \eta\left(\Phi_{11}+\Phi_{21} \frac{S_{2}}{S_{1}}\right)^{2} \\
K_{1}=\frac{8 S_{1} L_{1}}{\rho_{0} c_{0}^{2} \pi^{2} S_{\mathrm{m}}^{2}} \frac{f_{1}^{2}}{f_{0}^{2}} k_{\mathrm{m}}\left(\Phi_{11}+\Phi_{21} \frac{S_{2}}{S_{1}}\right)^{2}, K_{3}=\frac{8 S_{1} L_{1} h_{\mathrm{m}}^{2}}{\rho_{0} c_{0}^{2} \pi^{2} S_{\mathrm{m}}^{2}} k_{3}\left(\Phi_{11}+\Phi_{21} \frac{S_{2}}{S_{1}}\right)^{2}
\end{gathered}
$$

and

$$
\tilde{p}_{\mathrm{s}}(\tau)=E_{1} \cos \left(\tilde{\omega}_{1}^{\mathrm{s}} \tau+\varphi_{1}^{\mathrm{s}}\right)+E_{2} \cos \left(\tilde{\omega}_{2}^{\mathrm{S}} \tau+\varphi_{2}^{\mathrm{s}}\right)
$$

with

$$
\tilde{\omega}_{1}^{\mathrm{s}}=\frac{\omega_{1}^{\mathrm{s}}}{\omega_{1}^{\mathrm{p}}} \text { and } \tilde{\omega}_{2}^{\mathrm{s}}=\frac{\omega_{2}^{\mathrm{s}}}{\omega_{1}^{\mathrm{p}}}
$$

Note that now dot $(\cdot)$ denotes the differentiation with respect to the nondimensional time $\tau$.

To simplify the notations, the upper symbol tilde $(\sim)$ will be dropped in the sequel and the time dependence will be omitted.

The time normalization has been defined from the resonance frequency $\omega_{1}^{\mathrm{p}}$ of the pipe 1 (see Eq. (2)). This choice gives closed-form expressions for the nondimensional model parameters and hence facilitated the analysis of the order of magnitude of these parameters (see section below). Of course, the resonance frequency $\omega_{1}^{\mathrm{a}}$ of the acoustics part (see Eq. (17)) could also be used. Note that in the configuration under interest, $\omega_{1}^{\mathrm{a}}$ and $\omega_{1}^{\mathrm{p}}$ are very close (see Table 1). 


\subsection{About the order of magnitude of the parameters}

The parameters in Eqs. (24-26) have not the same order of magnitude. Firstly, to ensure that the membrane can be viewed as a grounded NES [5] with respect to the acoustic medium, the volume of the coupling box has to be chosen large enough with respect to the pipe volumes. These choices imply that the volume ratios are small $(\approx \epsilon<<1)$ and hence, following $(29)$ and (31), $\beta_{1}$ and $\beta_{2}$ are proportional to $\epsilon$. Considering now the nonlinear term $K_{3}$, due to the scaling process (22) and (23) the order of magnitude of $K_{3}$ (see (34)) is given by $\left(h_{\mathrm{m}} / R_{\mathrm{m}}\right)^{2} h_{\mathrm{m}}$ which leads to a parameter of order $\epsilon$ if $h_{\mathrm{m}}<<1$ and $h_{\mathrm{m}}<<R_{\mathrm{m}}$. Finally, the damping parameters $\lambda_{11}, \lambda_{12}, \lambda_{21}$, $\lambda_{22}, C_{1}$ and $C_{3}$ which model the acoustical and material (in the membrane) dissipative phenomena can also be considered as parameters of order $\epsilon$.

These properties will be used in the sequel to study analytically the equations of motion (24-26).

\section{Analytic treatment}

This section is devoted to the analytical study of quasi-periodic regimes when the frequencies of excitation are near the two resonance frequencies of the system.

The complexification method combined to the averaging method will be applied starting from Eqs. (24-26) written as

$$
\begin{aligned}
\ddot{x}_{1}+\lambda_{11}^{\epsilon} \dot{x}_{1}+\lambda_{12}^{\epsilon} \dot{x}_{2}+\omega_{1}^{2} x_{1}-\beta_{1}^{\epsilon} x_{3} & =-F_{1} p_{\mathrm{s}}, \\
\ddot{x}_{2}+\lambda_{21}^{\epsilon} \dot{x}_{1}+\lambda_{22}^{\epsilon} \dot{x}_{2}+\omega_{2}^{2} x_{2}-\beta_{2}^{\epsilon} x_{3} & =-F_{2} p_{\mathrm{s}}, \\
\gamma \ddot{x}_{3}+C_{1}^{\epsilon} \dot{x}_{3}+C_{3}^{\epsilon} x_{3}^{2} \dot{x}_{3}+K_{1} x_{3}+K_{3}^{\epsilon} x_{3}^{3} & \\
+\beta_{3}^{\epsilon}\left(-x_{1}-x_{2}+x_{3}\right) & =0
\end{aligned}
$$

with

$$
p_{s}(\tau)=E_{1} \cos \left(\omega_{1}^{\mathrm{s}} \tau+\phi_{1}^{\mathrm{s}}\right)+E_{2} \cos \left(\omega_{2}^{\mathrm{s}} \tau+\phi_{2}^{\mathrm{s}}\right)
$$

where the upper script $(\epsilon)$ indicates the parameter is proportional to $\epsilon$. Choosing $\epsilon<<1$ establishes the order of magnitude of the corresponding parameters in agreement with the orders of magnitude discussed in Section 2.3.

We assume that the excitation frequencies are detuned off in the following form

$$
\omega_{1}^{\mathrm{s}}=\omega_{1}+\sigma_{1} \text { and } \omega_{2}^{\mathrm{s}}=\omega_{2}+\sigma_{2}
$$

where $\sigma_{1}$ and $\sigma_{2}$ are also considered as small parameters. 


\subsection{Complexified system}

New variables are introduced as

$$
x_{i}=x_{i}^{1}+x_{i}^{2} \text { for } i=1,2 \text { and } 3
$$

to capture frequency components with respect to $\omega_{1}$ and $\omega_{2}$ respectively. For $i=1,2$ and 3 , the following complex change of variables are considered

$$
\left\{\begin{aligned}
\varphi_{i}^{1} \mathrm{e}^{\mathrm{j} \omega_{1} \tau} & =\frac{\dot{x}_{i}^{1}}{\omega_{1}}+\mathrm{j} x_{i}^{1} \\
\varphi_{i}^{2} \mathrm{e}^{\mathrm{j} \omega_{2} \tau} & =\frac{\dot{x}_{i}^{2}}{\omega_{2}}+\mathrm{j} x_{i}^{2}
\end{aligned}\right.
$$

where $\mathrm{j}=\sqrt{-1}$. The variables $\varphi_{i}^{1}$ and $\varphi_{i}^{2}$ are assumed as slowly evolving compared to the frequencies of excitation.

Substituting Eq. (43) into Eq. (42), we obtain

$$
x_{i}=-\frac{\mathrm{j}}{2}\left(\varphi_{i}^{1} \mathrm{e}^{\mathrm{j} \omega_{1} \tau}-\overline{\varphi_{i}^{1}} \mathrm{e}^{-\mathrm{j} \omega_{1} \tau}+\varphi_{i}^{2} \mathrm{e}^{\mathrm{j} \omega_{2} \tau}-\overline{\varphi_{i}^{2}} \mathrm{e}^{-\mathrm{j} \omega_{2} \tau}\right)
$$

and after time derivation

$$
\begin{aligned}
\dot{x}_{i}= & \frac{\omega_{1}}{2}\left(\varphi_{i}^{1} \mathrm{e}^{\mathrm{j} \omega_{1} \tau}+\overline{\varphi_{i}^{1}} \mathrm{e}^{-\mathrm{j} \omega_{1} \tau}\right)+\frac{\omega_{2}}{2}\left(\varphi_{i}^{2} \mathrm{e}^{\mathrm{j} \omega_{2} \tau}+\overline{\varphi_{i}^{2}} \mathrm{e}^{-\mathrm{j} \omega_{2} \tau}\right), \\
\ddot{x}_{i}= & \omega_{1}\left(\dot{\varphi}_{i}^{1} \mathrm{e}^{\mathrm{j} \omega_{1} \tau}+\mathrm{j} \omega_{1} \varphi_{i}^{1} \mathrm{e}^{\mathrm{j} \omega_{1} \tau}\right)+\omega_{2}\left(\dot{\varphi}_{i}^{2} \mathrm{e}^{\mathrm{j} \omega_{2} \tau}+\mathrm{j} \omega_{2} \varphi_{i}^{2} \mathrm{e}^{\mathrm{j} \omega_{2} \tau}\right) \\
& -\frac{\mathrm{j}}{2} \omega_{1}^{2}\left(\varphi_{i}^{1} \mathrm{e}^{\mathrm{j} \omega_{1} \tau}+\overline{\varphi_{i}^{1}} \mathrm{e}^{-\mathrm{j} \omega_{1} \tau}\right)-\frac{\mathrm{j}}{2} \omega_{2}^{2}\left(\varphi_{i}^{2} \mathrm{e}^{\mathrm{j} \omega_{2} \tau}+\overline{\varphi_{i}^{2}} \mathrm{e}^{-\mathrm{j} \omega_{2} \tau}\right)
\end{aligned}
$$

where $\left(^{-}\right)$denotes complex conjugate.

As usual in multi-periodic case[14, 15], a multiple time parameter $\left(\boldsymbol{\tau}_{1}, \boldsymbol{\tau}_{2}\right)$ can be introduced

$$
\left(\boldsymbol{\tau}_{1}, \boldsymbol{\tau}_{2}\right)=\left(\omega_{1} \tau, \omega_{2} \tau\right)
$$

giving

$$
x_{i}=-\frac{\mathrm{j}}{2}\left(\varphi_{i}^{1} \mathrm{e}^{\mathrm{j} \tau_{1}}-\overline{\varphi_{i}^{1}} \mathrm{e}^{-\mathrm{j} \tau_{1}}+\varphi_{i}^{2} \mathrm{e}^{\mathrm{j} \tau_{2}}-\overline{\varphi_{i}^{2}} \mathrm{e}^{-\mathrm{j} \tau_{2}}\right) \text { for } i=1,2 \text { and } 3 .
$$

Substituting Eqs. (44-46) into Eqs. (37-39), the resulting equations can be averaged with respect to the excitation frequencies $\omega_{1}$ and $\omega_{2}$ separately 
yielding the following system of slow modulation

$$
\begin{aligned}
\omega_{1} \dot{\varphi}_{1}^{1}+\frac{\lambda_{11}^{\epsilon} \omega_{1}}{2} \varphi_{1}^{1}+\frac{\lambda_{12}^{\epsilon} \omega_{1}}{2} \varphi_{2}^{1}+\mathrm{j} \frac{\beta_{1}^{\epsilon}}{2} \varphi_{3}^{1} & =-\frac{F_{1} E_{1}}{2} \mathrm{e}^{\mathrm{j}\left(\sigma_{1} \tau+\phi_{1}^{\mathrm{S}}\right)}, \\
\omega_{1} \dot{\varphi}_{2}^{1}+\frac{\lambda_{21}^{\epsilon} \omega_{1}}{2} \varphi_{1}^{1}+\left(\frac{\lambda_{22}^{\epsilon} \omega_{1}}{2}+\frac{\mathrm{j}}{2}\left(\omega_{1}^{2}-\omega_{2}^{2}\right)\right) \varphi_{2}^{1} & +\mathrm{j} \frac{\beta_{2}^{\epsilon}}{2} \varphi_{3}^{1}=-\frac{F_{2} E_{1}}{2} \mathrm{e}^{\mathrm{j}\left(\sigma_{1} \tau+\phi_{1}^{\mathrm{S}}\right)}, \\
\gamma \omega_{1} \dot{\varphi}_{3}^{1}+\left(\frac{C_{1}^{\epsilon} \omega_{1}}{2}+\frac{\mathrm{j}}{2}\left(\gamma \omega_{1}^{2}-K_{1}-\beta_{3}^{\epsilon}\right)\right) \varphi_{3}^{1} & \\
+\left(\frac{C_{3}^{\epsilon} \omega_{1}}{8}-\mathrm{j} \frac{3 K_{3}^{\epsilon}}{8}\right)\left(\left|\varphi_{3}^{1}\right|^{2}+2\left|\varphi_{3}^{2}\right|^{2}\right) \varphi_{3}^{1} & \\
+\mathrm{j} \frac{\beta_{3}^{\epsilon}}{2}\left(\varphi_{1}^{1}+\varphi_{2}^{1}\right) & =0 \\
\omega_{2} \dot{\varphi}_{1}^{2}+\left(\frac{\lambda_{11}^{\epsilon} \omega_{2}}{2}+\frac{\mathrm{j}}{2}\left(\omega_{2}^{2}-\omega_{1}^{2}\right)\right) \varphi_{1}^{2}+\frac{\lambda_{12}^{\epsilon} \omega_{2}}{2} \varphi_{2}^{2} & \\
+\mathrm{j} \frac{\beta_{1}^{\epsilon}}{2} \varphi_{3}^{2} & =-\frac{F_{1} E_{2}}{2} \mathrm{e}^{\mathrm{j}\left(\sigma_{2} \tau+\phi_{2}^{\mathrm{S}}\right)}, \\
\omega_{2} \dot{\varphi}_{2}^{2}+\frac{\lambda_{21}^{\epsilon} \omega_{2}}{2} \varphi_{1}^{2}+\frac{\lambda_{22}^{\epsilon} \omega_{2}}{2} \varphi_{2}^{2}+\frac{\mathrm{j}}{2} \beta_{2}^{\epsilon} \varphi_{3}^{2} & =-\frac{F_{2} E_{2}}{2} \mathrm{e}^{\mathrm{j}\left(\sigma_{2} \tau+\phi_{2}^{\mathrm{S}}\right)}, \\
\gamma \omega_{2} \dot{\varphi}_{3}^{2}+\left(\frac{C_{1}^{\epsilon} \omega_{2}}{2}+\frac{\mathrm{j}}{2}\left(\gamma \omega_{2}^{2}-K_{1}-\beta_{3}^{\epsilon}\right)\right) \varphi_{3}^{2} & \\
+\left(\frac{C_{3}^{\epsilon} \omega_{2}}{8}-\mathrm{j} \frac{3 K_{3}^{\epsilon}}{8}\right)\left(\left|\varphi_{3}^{2}\right|^{2}+2\left|\varphi_{3}^{1}\right|^{2}\right) \varphi_{3}^{2} & \\
+\mathrm{j} \frac{\beta_{3}^{\epsilon}}{2}\left(\varphi_{1}^{2}+\varphi_{2}^{2}\right) & =0 .
\end{aligned}
$$

The first three equations Eqs (49)-(51) have been obtained using the following averaging operator

$$
\int_{0}^{2 \pi} \int_{0}^{2 \pi} R\left(\boldsymbol{\tau}_{1}, \boldsymbol{\tau}_{2}\right) \mathrm{e}^{-i \boldsymbol{\tau}_{1}} \mathrm{~d} \boldsymbol{\tau}_{1} \mathrm{~d} \boldsymbol{\tau}_{2}
$$

written in multi-time parameter. The last three equations Eqs (52)-(54) result from the use of the following averaging operator

$$
\int_{0}^{2 \pi} \int_{0}^{2 \pi} R\left(\boldsymbol{\tau}_{1}, \boldsymbol{\tau}_{2}\right) \mathrm{e}^{-i \boldsymbol{\tau}_{2}} \mathrm{~d} \boldsymbol{\tau}_{1} \mathrm{~d} \boldsymbol{\tau}_{2} .
$$

An additional change of variables is needed to reduce the system into autonomous one. 
Introducing for $i=1,2$ and 3 , the following new variables

$$
\left\{\begin{array}{l}
\hat{\varphi}_{i}^{1}=\varphi_{i}^{1} \mathrm{e}^{-\mathrm{j} \sigma_{1} \tau-\mathrm{j} \phi_{1}^{\mathrm{s}}} \\
\hat{\varphi}_{i}^{2}=\varphi_{i}^{2} \mathrm{e}^{-\mathrm{j} \sigma_{2} \tau-\mathrm{j} \phi_{2}^{\mathrm{s}}}
\end{array},\right.
$$

Eqs (49-54) are reduced to the autonomous form

$$
\begin{aligned}
\omega_{1} \dot{\varphi}_{1}^{1}+\left(\frac{\lambda_{11}^{\epsilon} \omega_{1}}{2}+\frac{\mathrm{j}}{2} 2 \omega_{1} \sigma_{1}\right) \varphi_{1}^{1}+\frac{\lambda_{12}^{\epsilon} \omega_{1}}{2} \varphi_{2}^{1} & +\mathrm{j} \frac{\beta_{1}^{\epsilon}}{2} \varphi_{3}^{1}=-\frac{F_{1} E_{1}}{2}, \\
\omega_{1} \dot{\varphi}_{2}^{1}+\frac{\lambda_{21}^{\epsilon} \omega_{1}}{2} \varphi_{1}^{1}+\left(\frac{\lambda_{22}^{\epsilon} \omega_{1}}{2}+\frac{\mathrm{j}}{2}\left(2 \omega_{1} \sigma_{1}+\omega_{1}^{2}-\omega_{2}^{2}\right)\right) \varphi_{2}^{1} & +\mathrm{j} \frac{\beta_{2}^{\epsilon}}{2} \varphi_{3}^{1}=-\frac{F_{2} E_{1}}{2}, \\
\gamma \omega_{1} \dot{\varphi}_{3}^{1}+\left(\frac{C_{1}^{\epsilon} \omega_{1}}{2}+\frac{\mathrm{j}}{2}\left(2 \gamma \omega_{1} \sigma_{1}+\gamma \omega_{1}^{2}-K_{1}-\beta_{3}^{\epsilon}\right)\right) \varphi_{3}^{1} & =0 \\
+\left(\frac{C_{3}^{\epsilon} \omega_{1}}{8}-\mathrm{j} \frac{3 K_{3}^{\epsilon}}{8}\right)\left(\left|\varphi_{3}^{1}\right|^{2}+2\left|\varphi_{3}^{2}\right|^{2}\right) \varphi_{3}^{1}+\mathrm{j} \frac{\beta_{3}^{\epsilon}}{2}\left(\varphi_{1}^{1}+\varphi_{2}^{1}\right) & =0 \\
\omega_{2} \dot{\varphi}_{1}^{2}+\left(\frac{\lambda_{11}^{\epsilon} \omega_{2}}{2}+\frac{\mathrm{j}}{2}\left(2 \omega_{2} \sigma_{2}+\omega_{2}^{2}-\omega_{1}^{2}\right)\right) \varphi_{1}^{2}+\frac{\lambda_{12}^{\epsilon} \omega_{2}}{2} \varphi_{2}^{2} & =-\frac{F_{1} E_{2}}{2}, \\
\omega_{2} \dot{\varphi}_{2}^{2}+\frac{\lambda_{21}^{\epsilon} \omega_{2}}{2} \varphi_{1}^{2}+\left(\frac{\lambda_{22}^{\epsilon} \omega_{2}}{2}+\frac{\mathrm{j}}{2} 2 \omega_{2} \sigma_{2}\right) \varphi_{2}^{2} & \\
+\mathrm{j} \frac{\beta_{2}^{\epsilon}}{2} \varphi_{3}^{2} & =-\frac{F_{2} E_{2}}{2}, \\
+\left(\frac{C_{3}^{\epsilon} \omega_{2}}{8}-\mathrm{j} \frac{3 K_{3}^{\epsilon}}{8}\right)\left(\left|\varphi_{3}^{2}\right|^{2}+2\left|\varphi_{3}^{1}\right|^{2}\right) \varphi_{3}^{2}+\mathrm{j} \frac{\beta_{3}^{\epsilon}}{2}\left(\varphi_{1}^{2}+\varphi_{2}^{2}\right) & =0 . \\
\omega_{3}^{2}+\left(\frac{C_{1}^{\epsilon} \omega_{2}}{2}+\frac{\mathrm{j}}{2}\left(2 \gamma \omega_{2} \sigma_{2}+\gamma \omega_{2}^{2}-K_{1}-\beta_{3}^{\epsilon}\right)\right) \varphi_{3}^{2} &
\end{aligned}
$$

To simplify the hat sign $\left({ }^{\wedge}\right)$ has been omitted.

When $E_{2}=0$ (respectively $E_{1}=0$ ), Eqs. (61-63) (respectively Eqs. (5860)) are trivially satisfied with $\varphi_{1}^{2}=\varphi_{2}^{2}=\varphi_{3}^{2}=0$ (respectively $\varphi_{1}^{1}=\varphi_{2}^{1}=$ $\left.\varphi_{3}^{1}=0\right)$ and the resulting equations Eqs. (58-60) (respectively Eqs. (61-63)) are in agreement with the results described in [12].

\subsection{Quasi-periodic solutions}

The quasi-periodic solutions of Eqs. (37-39) correspond to the fixed points

$$
\boldsymbol{\varphi}_{0}=\left(\varphi_{10}^{1}, \varphi_{20}^{1}, \varphi_{30}^{1}, \varphi_{10}^{2}, \varphi_{20}^{2}, \varphi_{30}^{2}\right)^{T}
$$


of Eqs (58-63).

By setting

$$
\dot{\varphi}_{1}^{1}=\dot{\varphi}_{1}^{2}=\dot{\varphi}_{2}^{1}=\dot{\varphi}_{2}^{2}=\dot{\varphi}_{3}^{1}=\dot{\varphi}_{3}^{2}=0,
$$

we obtain a system of algebraic equations which can be re-organized as

$$
\begin{aligned}
& \left(\frac{\lambda_{11}^{\epsilon} \omega_{1}}{2}+\frac{\mathrm{j}}{2} 2 \omega_{1} \sigma_{1}\right) \varphi_{10}^{1}+\frac{\lambda_{12}^{\epsilon} \omega_{1}}{2} \varphi_{20}^{1}=-\mathrm{j} \frac{\beta_{1}^{\epsilon}}{2} \varphi_{30}^{1}-\frac{F_{1} E_{1}}{2}, \\
& \frac{\lambda_{21}^{\epsilon} \omega_{1}}{2} \varphi_{10}^{1}+\left(\frac{\lambda_{22}^{\epsilon} \omega_{1}}{2}+\frac{\mathrm{j}}{2}\left(2 \omega_{1} \sigma_{1}+\omega_{1}^{2}-\omega_{2}^{2}\right)\right) \varphi_{20}^{1}=-\mathrm{j} \frac{\beta_{2}^{\epsilon}}{2} \varphi_{30}^{1}-\frac{F_{2} E_{1}}{2}, \\
& \left(\frac{C_{1}^{\epsilon} \omega_{1}}{2}+\frac{\mathrm{j}}{2}\left(2 \gamma \omega_{1} \sigma_{1}+\gamma \omega_{1}^{2}-K_{1}-\beta_{3}^{\epsilon}\right)\right) \varphi_{30}^{1} \\
& +\left(\frac{C_{3}^{\epsilon} \omega_{1}}{8}-\mathrm{j} \frac{3 K_{3}^{\epsilon}}{8}\right)\left(\left|\varphi_{30}^{1}\right|^{2}+2\left|\varphi_{30}^{2}\right|^{2}\right) \varphi_{30}^{1}=-\mathrm{j} \frac{\beta_{3}^{\epsilon}}{2}\left(\varphi_{10}^{1}+\varphi_{20}^{1}\right), \\
& \left(\frac{\lambda_{11}^{\epsilon} \omega_{2}}{2}+\frac{\mathrm{j}}{2}\left(2 \omega_{2} \sigma_{2}+\omega_{2}^{2}-\omega_{1}^{2}\right)\right) \varphi_{10}^{2}+\frac{\lambda_{12}^{\epsilon} \omega_{2}}{2} \varphi_{20}^{2}=-\mathrm{j} \frac{\beta_{1}^{\epsilon}}{2} \varphi_{30}^{2}-\frac{F_{1} E_{2}}{2}, \\
& \frac{\lambda_{21}^{\epsilon} \omega_{2}}{2} \varphi_{10}^{2}+\left(\frac{\lambda_{2}^{\epsilon} \omega_{2}}{2}+\frac{\mathrm{j}}{2} 2 \omega_{2} \sigma_{2}\right) \varphi_{20}^{2}=-\mathrm{j} \frac{\beta_{2}^{\epsilon}}{2} \varphi_{30}^{2}-\frac{F_{2} E_{2}}{2} \\
& \left(\frac{C_{1}^{\epsilon} \omega_{2}}{2}+\frac{\mathrm{j}}{2}\left(2 \omega_{2} \gamma \sigma_{2}+\gamma \omega_{2}^{2}-K_{1}-\beta_{3}^{\epsilon}\right)\right) \varphi_{30}^{2} \\
& +\left(\frac{C_{3}^{\epsilon} \omega_{2}}{8}-\mathrm{j} \frac{3 K_{3}^{\epsilon}}{8}\right)\left(\left|\varphi_{30}^{2}\right|^{2}+2\left|\varphi_{30}^{1}\right|^{2}\right) \varphi_{30}^{2}=-\mathrm{j} \frac{\beta_{3}^{\epsilon}}{2}\left(\varphi_{10}^{2}+\varphi_{20}^{2}\right) \text {. }
\end{aligned}
$$

Solving the linear system Eqs. (66-67) (respectively Eqs. (69-70)) with respect to the unknown variables $\varphi_{10}^{1}$ and $\varphi_{20}^{1}$ (respectively $\varphi_{10}^{2}$ and $\varphi_{20}^{2}$ ) and substituting the result into in Eq. (68) (respectively Eq. (71)), we obtain

$$
\varphi_{30}^{1}\left(b_{0}+b_{1}\left|\varphi_{30}^{1}\right|^{2}+b_{2}\left|\varphi_{30}^{2}\right|^{2}\right)=c_{0}
$$

(respectively

$$
\left.\varphi_{30}^{2}\left(d_{0}+d_{1}\left|\varphi_{30}^{1}\right|^{2}+d_{2}\left|\varphi_{30}^{2}\right|^{2}\right)=e_{0}\right)
$$

where $b_{0}, b_{1}, b_{2}, c_{0}, d_{0}, d_{1}, d_{2}$ and $e_{0}$ are complex coefficients (not given here). Finally Eqs. (72-73) can be reduced to the following two polynomials of order 3 in $Z_{1}=\left|\varphi_{30}^{1}\right|^{2}$ and $Z_{2}=\left|\varphi_{30}^{2}\right|^{2}$ with real coefficients

$$
\begin{gathered}
b_{1} \overline{b_{1}} Z_{1}^{3}+\left(b_{1} \overline{b_{2}}+\overline{b_{1}} b_{2}\right) Z_{1}^{2} Z_{2}+b_{2} \overline{b_{2}} Z_{1} Z_{2}^{2}+ \\
\left(b_{0} \overline{b_{1}}+\overline{b_{0}} b_{1}\right) Z_{1}^{2}+\left(b_{0} \overline{b_{2}}+\overline{b_{0}} b_{2}\right) Z_{1} Z_{2}+b_{0} \overline{b_{0}} Z_{1}=c_{0} \overline{c_{0}} \\
d_{2} \overline{d_{2}} Z_{2}^{3}+\left(d_{1} \overline{d_{2}}+\overline{d_{1}} d_{2}\right) Z_{2}^{2} Z_{1}+d_{1} \overline{d_{1}} Z_{1} Z_{2}^{2}+ \\
\left(d_{0} \overline{d_{2}}+\overline{d_{0}} d_{2}\right) Z_{2}^{2}+\left(d_{0} \overline{d_{1}}+\overline{d_{0}} d_{1}\right) Z_{1} Z_{2}+d_{0} \overline{d_{0}} Z_{2}=e_{0} \overline{e_{0}} .
\end{gathered}
$$


The polynomial system $(74)(75)$ admits at most 9 zeros $Z_{0}=\left(Z_{10}, Z_{20}\right)$. The zeros can be real-real, real-complex, or complex-complex moreover nonreal terms occur in complex conjugate pair of zeros. The quasi-periodic solutions correspond to real-real zeros with both positive values. Note that starting from $Z_{10}=\left|\varphi_{30}^{1}\right|^{2}$ and $Z_{20}=\left|\varphi_{30}^{2}\right|^{2}, \varphi_{10}^{1}, \varphi_{20}^{1}$ and $\varphi_{30}^{1}$ (respectively $\varphi_{10}^{2}, \varphi_{20}^{2}$ and $\left.\varphi_{30}^{2}\right)$ can easily be deduced from Eqs. (72) and (66-67) (respectively Eqs. (73) and (69-71)).

\subsection{Stability analysis and local bifurcation of the quasi-periodic solutions}

The stability analysis of a quasi-periodic response of Eqs. (37-39) can be explored analyzing the stability of the associated fixed point $\varphi_{0}$ of Eqs. (58$63)$.

Re-writing Eqs. (58-63) as

$$
\dot{\varphi}=\mathrm{A} \varphi+\mathrm{B}(\varphi, \bar{\varphi})
$$

where $\boldsymbol{\varphi}=\left(\varphi_{1}^{1}, \varphi_{2}^{1}, \varphi_{3}^{1}, \varphi_{1}^{2}, \varphi_{2}^{2}, \varphi_{3}^{2}\right)^{T}$, introducing the linearized terms of $\mathbf{B}$ and its conjugate $\overline{\mathbf{B}}$ around $(\boldsymbol{\varphi}, \bar{\varphi})=\left(\varphi_{0}, \overline{\varphi_{0}}\right)$ as

$$
\begin{aligned}
& \mathbf{B}\left(\varphi_{0}+\boldsymbol{\delta} \boldsymbol{\varphi}, \overline{\varphi_{0}}+\overline{\boldsymbol{\delta} \varphi}\right) \approx \partial_{\boldsymbol{\varphi}} \mathbf{B}\left(\varphi_{0}, \overline{\varphi_{0}}\right) \boldsymbol{\delta} \boldsymbol{\varphi}+\partial_{\bar{\varphi}} \mathbf{B}\left(\varphi_{0}, \overline{\varphi_{0}}\right) \overline{\boldsymbol{\delta} \boldsymbol{\varphi}} \\
& \overline{\mathbf{B}}\left(\varphi_{0}+\boldsymbol{\delta} \boldsymbol{\varphi}, \overline{\varphi_{0}}+\overline{\boldsymbol{\delta} \varphi}\right) \approx \partial_{\boldsymbol{\varphi}} \overline{\mathbf{B}}\left(\varphi_{0}, \overline{\varphi_{0}}\right) \boldsymbol{\delta} \varphi+\partial_{\bar{\varphi}} \overline{\mathbf{B}}\left(\varphi_{0}, \overline{\varphi_{0}}\right) \overline{\boldsymbol{\delta} \varphi}
\end{aligned}
$$

and linearizing Eq. (76) (and its conjugate equation) at $(\boldsymbol{\varphi}, \overline{\boldsymbol{\varphi}})=\left(\boldsymbol{\varphi}_{0}, \overline{\boldsymbol{\varphi}_{0}}\right)$, we obtain the following close linear system

$$
\left(\begin{array}{c}
\dot{\boldsymbol{\delta} \varphi} \\
\dot{\boldsymbol{\delta} \varphi}
\end{array}\right)=\left(\begin{array}{cc}
\left.\mathbf{A}+\partial_{\varphi} \mathbf{B}\left(\varphi_{0}, \overline{\varphi_{0}}\right)\right) & \partial_{\bar{\varphi}} \mathbf{B}\left(\varphi_{0}, \overline{\varphi_{0}}\right) \\
\partial_{\varphi} \overline{\mathbf{B}}\left(\varphi_{0}, \overline{\varphi_{0}}\right) & \bar{A}+\partial_{\bar{\varphi}} \overline{\mathbf{B}}\left(\boldsymbol{\varphi}_{0}, \overline{\varphi_{0}}\right)
\end{array}\right)\left(\begin{array}{c}
\boldsymbol{\delta} \boldsymbol{\varphi} \\
\overline{\boldsymbol{\delta} \varphi}
\end{array}\right) .
$$

The eigenvalues of the associated matrix characterize the local stability property of the fixed point $\boldsymbol{\varphi}_{0}$. The eigenvalues can also be used to localize bifurcation points with respect to some control parameters. Saddle-Node $(\mathrm{SN})$ and Hopf bifurcations will be analyzed in the sequel with respect to the detuning frequency parameters $\sigma_{1}$ and $\sigma_{2}$.

\section{Application to a nominal configuration}

In view of future experiments, the vibro-acoustic system (see Fig. 1) under study is defined from the numerical values of the parameters given in terms of geometrical quantities $L_{1}=2.40 \mathrm{~m}, d_{1}=2 \times 0.075 \mathrm{~m}, S_{1}=\pi \times 0.075^{2} \mathrm{~m}^{2}$, 
$L_{2}=1.60 \mathrm{~m}, d_{2}=2 \times 0.10 \mathrm{~m}, S_{2}=\pi \times 0.10^{2} \mathrm{~m}^{2}, V_{\mathrm{m}}=2 \times 0.027 \mathrm{~m}^{3}, d_{\mathrm{m}}=2 \times$ $0.03 \mathrm{~m}, S_{\mathrm{m}}=\pi 0.03^{2} \mathrm{~m}^{2}, h_{\mathrm{m}}=0.3 \times 0.001 \mathrm{~m}$; in terms of material quantities $\rho_{0}=1.3 \mathrm{~kg} \mathrm{~m}^{-3}, c_{0}=350 \mathrm{~m} \mathrm{~s}^{-1}, \rho_{\mathrm{m}}=980 \mathrm{~kg} \mathrm{~m}^{-3}, E=1480000 \mathrm{~Pa}$ and $\nu=0.49$ and in terms of damping quantities $\tau_{1}=0.007$ and $\tau_{2}=0.007$. With this choice, the volume $S_{\mathrm{m}}$ of the coupling box is larger than the pipe volumes $L_{1} S_{1}$ and $L_{2} S_{2}$.

The associated numerical values of the parameters characterizing the dimensional model (see Eqs (10-12)) are then given by $m_{1}=0.0275675 \mathrm{~kg}, k_{1}=$ $5786.43 \mathrm{~N} \mathrm{~m}^{-1}, m_{2}=0.0326726 \mathrm{~kg}, k_{2}=15430.5 \mathrm{~N} \mathrm{~m}^{-1}, k_{b}=2.949 \times 10^{6}$, $m_{m}=0.000277088 \mathrm{~kg}, k_{m}=0.527154 \mathrm{~N} \mathrm{~m}^{-1}, k_{3}=5.43879 \times 10^{6} \mathrm{~N} \mathrm{~m}^{-3}$, $\eta=0.00025, f_{0}=6.94192 \mathrm{~Hz}$ and $f_{1}=40 \mathrm{~Hz}$. Note that the values of the parameters related to the membrane $\left(m_{m}, k_{m}, k_{3}, \eta, f_{0}\right.$ and $\left.f_{1}\right)$ have been chosen in reference to the experiment data given in [2].

This set of parameter values is in agreement with the order of magnitude of the parameters discussed in Section 2.3. The numerical values of the parameters characterizing the nondimensional model (see Eqs. (24-26)) follow as $\omega_{1}=1.05632, \beta_{1}=0.0819989, F_{1}=0.06556, \omega_{2}=1.64101, \beta_{2}=0.501569$, $F_{2}=0.401016, \gamma=0.809151, K_{1}=0.243498, K_{3}=0.00680993, C_{1}=$ $0.000840005, C_{3}=0.00155998, \lambda_{11}=0.0141894, \lambda_{12}=-0.000459194, \lambda_{21}=$ -0.00280879 and $\lambda_{22}=0.0119723$.

\begin{tabular}{c|c|c|c|c}
\hline$i$ & $\omega_{i}^{\mathrm{p}}$ & $\omega_{i}^{\mathrm{a}}$ & $\omega_{i}^{\mathrm{va}}$ & $\omega_{i}=\frac{\omega_{i}^{a}}{\omega_{1}^{p}}$ \\
& $(\mathrm{~Hz})$ & $(\mathrm{Hz})$ & $(\mathrm{Hz})$ & \\
\hline 1 & 458.149 & 483.953 & 486.329 & 1.05632 \\
& $(72.9167)$ & $(77.023)$ & $(77.402)$ & \\
\hline 2 & 687.223 & 755.286 & 758.309 & 1.64856 \\
& $(109.375)$ & $(120.208)$ & $(120.689)$ & \\
\hline
\end{tabular}

Table 1: Resonance frequencies.

The values of the resonance frequencies $\omega_{i}^{\mathrm{p}}$ (as defined in Eqs. (2) and (4)) and $\omega_{i}^{\mathrm{a}}$ (as defined in Eq. (17)) are given in Table 1 and compared with the resonance frequencies $\omega_{i}^{\mathrm{va}}$ of the underlying linear system $\left(k_{3}=0\right)$ of the dimensional model Eqs. (10-12). Also reported in Table 1 are the values of the reduced resonance frequencies $\omega_{i}$ characterizing Eqs. (37-39). As already mentioned, $\omega_{1}^{\mathrm{p}}$ and $\omega_{1}^{\mathrm{a}}$ are close. Moreover $\omega_{i}^{\mathrm{a}}$ and $\omega_{i}^{\mathrm{va}}$ are also close showing that the linear part of the membrane does not introduce a strong coupling 
between the pipes and the membrane. The resonance frequencies of the acoustics medium are well separated.

Here, it is interesting to note that choosing the detuning parameters $\sigma_{1}$ and $\sigma_{2}$ as $\frac{\sigma_{1}}{\omega_{1}}=0$ and $\frac{\sigma_{2}}{\omega_{2}}=0$, the excitation frequencies of the associated bi-periodic excitation are equal to the resonance frequencies $\omega_{i}^{\mathrm{p}}$. Moreover choosing the detuning parameters $\sigma_{1}$ and $\sigma_{2}$ as $\frac{\sigma_{1}}{\omega_{1}}=\frac{\omega_{i}^{\mathrm{va}}}{\omega_{i}^{\mathrm{a}}}-1(\approx 0.0042)$ and $\frac{\sigma_{2}}{\omega_{2}}=\frac{\omega_{i}^{\mathrm{va}}}{\omega_{i}^{\mathrm{a}}}-1(\approx 0.004)$, the excitation frequencies of the associated bi-periodic excitation are equal to the resonance frequencies $\omega_{i}^{\mathrm{va}}$.

\subsection{Periodic solutions}

We assume here that the excitation is periodic with

$$
p_{s}(\tau)=E_{k} \cos \left(\omega_{k}^{\mathrm{s}} \tau\right) \text { with } E_{k}=e_{k} \frac{\pi \rho_{0} c_{0}^{2}}{V_{\mathrm{m}}} \times 10^{-6}
$$

for $k=1$ or 2 .

As already indicated in Section 3, the analytical treatment when the excitation is of the form (78) coincides with the methodology proposed in[12]. Hence we will just discuss some classical behaviors.

Fig. 2 shows the frequency-response diagrams deduced from the complexification approach combined to the averaging method. For the both cases $(k=1$ and $k=2)$, two excitation levels are considered: a low excitation level $\left(e_{1}=0.80\right.$ for $k=1$ and $e_{2}=1.60$ for $\left.k=2\right)$ giving one stable solution over the frequency range considered (see black curves in Fig. 2) and a high excitation level $\left(e_{1}=0.90\right.$ for $k=1$ and $e_{2}=1.80$ for $k=2$ ) showing no unique solution zones and instability properties (see grey curves in Fig. 2).

Considering now only the high excitation level cases $\left(e_{1}=0.90\right.$ for $k=1$ and $e_{2}=1.80$ for $k=2$ ), frequency-response diagrams in terms of $\max _{t \in\left[t_{1}, t_{2}\right]}\left|x_{i}(t)\right|$ for $i=1,2$ and 3 obtained from the fixed points of Eqs (58-63) and by numerical integration of Eqs. (37-39) using the fixed point as initial conditions $\left(t_{0}=0\right)$ are plotted Fig. 3. Also reported are the frequency-response diagrams obtained by numerical integration of the associated underlying linear system of Eqs. (37-39) (i.e. with $C_{3}=0$ and $K_{3}=0$ ). The solver NDSolve available in (C)Mathematica to solve ordinary differential equations was used with $t_{1}=1000$ and $t_{2}=2000$. The quantity $\max _{t \in\left[t_{1}, t_{2}\right]}\left|x_{i}(t)\right|$ was chosen because it can be used as a criterion for ear or structure damage. 

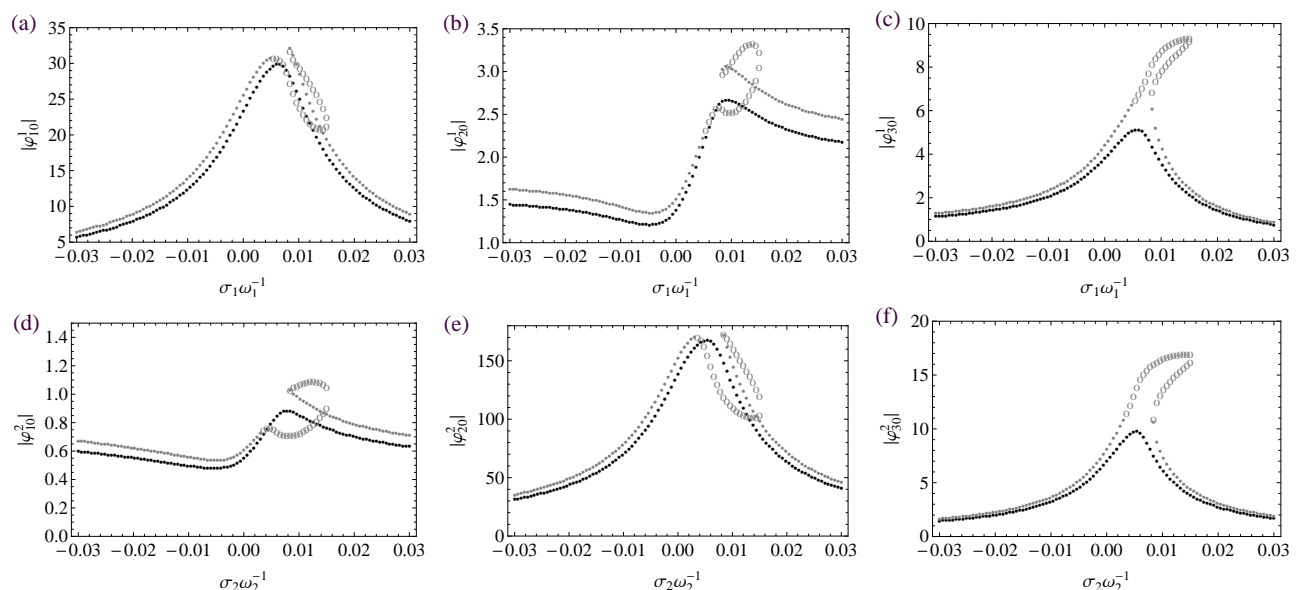

Figure 2: (a-c): $\left|\varphi_{i}^{10}\right|$ for $i=1,2$ and 3 with $e_{1}=0.80$ (black) and 0.90 (grey). (d-f): $\left|\varphi_{i}^{20}\right|$ for $i=1,2$ and 3 with $e_{2}=1.60$ (black) and 1.80 (grey). Stable solutions (dot markers), unstable solutions (circle markers). $\left(E_{k}=e_{k} \frac{\pi \rho_{0} c_{0}^{2}}{V_{\mathrm{m}}} \times 10^{-6}\right.$ for $k=1$ and 2$)$.

First of all, it interesting to note that the responses obtained by numerical integration of Eqs. (37-39) are very close to the responses predicted by the analytical method (fixed points of Eqs (58-63)) when the stability criterion is satisfied (see filled square markers in Fig. 3). Moreover, in the unstable zone, that is to say when no periodic solution exits, the responses obtained by numerical integration differ from the responses obtained by the analytical method. In this zone, the responses can be quasi-periodic or strongly quasiperiodic (see $[12,5])$. While a quasi-periodic solution can be found as a sum of periodic terms, a strongly quasi-periodic response, also named Strongly Modulated Response (SMR), can not be captured by a local (linear) analysis of the fixed point of the averaged equations. It is characterized by a magnitude of the amplitude modulation which is equal to the response amplitude (see the amplitude modulation Fig. 10)

As expected, when $k=1$ that is to say when the excitation frequency is near the resonance frequency $\omega_{1}$ (see Fig. 3(a-c)), the membrane acts as a nonlinear noise absorber. The $x_{1}$ component is reduced compared to the linear case. The energy is mainly transferred to the $x_{3}$ component with a smaller amplification of the $x_{2}$ component. Symmetrical results hold for $k=2$ (see Fig. 3(d-f)). 

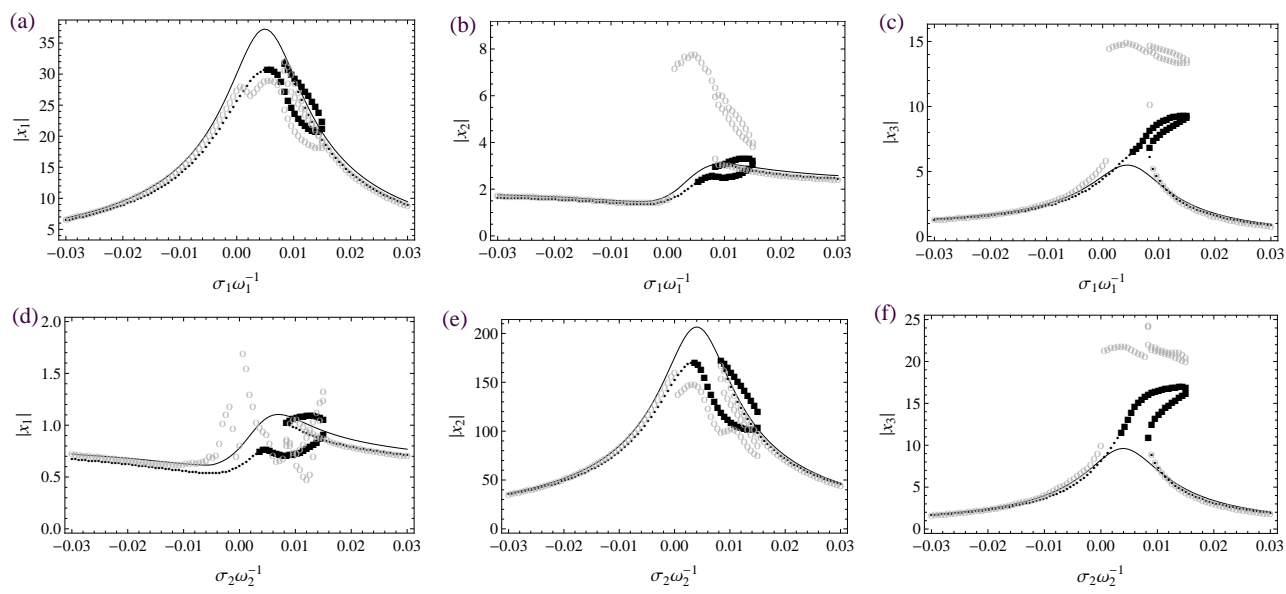

Figure 3: $\max _{t \in\left[t_{1}, t_{2}\right]}\left|x_{i}(t)\right|$ for $i=1,2$ and 3 obtained from the fixed points of Eqs (5863) (black), by numerical integration of Eqs. (37-39) (circle markers) and by numerical integration of the associated underlying linear system of Eqs. (37-39) (continuous curves). (a-c): $e_{1}=0.90$ and $e_{2}=0$. (d-f): $e_{1}=0$ and $e_{2}=1.80$. Filled square markers denote unstable fixed point solutions. $\left(E_{k}=e_{k} \frac{\pi \rho_{0} c_{0}^{2}}{V \mathrm{~m}} \times 10^{-6}\right.$ for $k=1$ and 2$)$.

\subsection{Quasi-periodic solutions}

We assume now that the excitation is quasi-periodic with

$$
p_{s}(\tau)=E_{1} \cos \left(\omega_{1}^{\mathrm{s}} \tau+\phi_{1}^{\mathrm{s}}\right)+E_{2} \cos \left(\omega_{2}^{\mathrm{s}} \tau+\phi_{2}^{\mathrm{s}}\right) \text { with } E_{k}=e_{k} \frac{\pi \rho_{0} c_{0}^{2}}{V_{\mathrm{m}}} \times 10^{-6}
$$

We first analyze the quasi-periodic responses from the complexification approach combined to the averaging method. The quasi-periodic responses are characterized from Eqs. (74-75). Fig. 4 shows the number of stationary solutions (fixed points of the complexified and averaged model) in the domain defined by $-0.3 \leq \sigma_{1} \omega_{1}^{-1} \leq 0.3$ and $-0.3 \leq \sigma_{2} \omega_{2}^{-1} \leq 0.3$ and for various excitations levels $\left(e_{1}, e_{2}\right)$ with $e_{1} \in\{0.80,0.90\}$ and $e_{2} \in\{1.60,1.80\}$. These level values were considered separately in the periodic case (Section 4.1). The stability zones are reported in Fig. 5. In terms of stability properties, Fig. 4 defines the boundary of the possible SN bifurcations whereas Fig. 5 defines the boundary of the possible Hopf bifurcations. Depending on the source detuning and amplitude, zones with one, three or five solutions have been found (see Fig. 4) associated to different stability properties (see Fig. 5). 

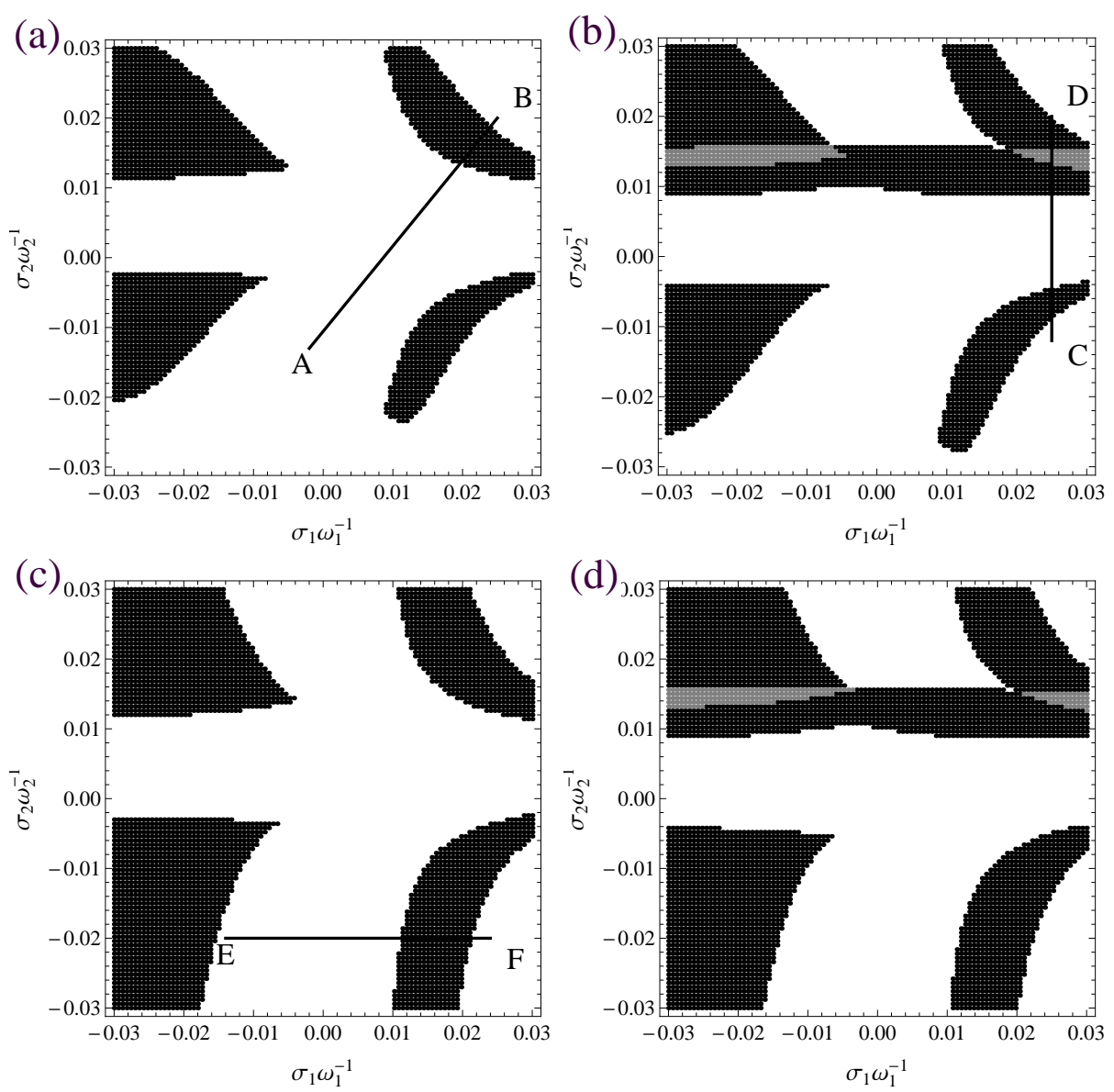

Figure 4: Number of fixed points of Eqs (58-63) for (a) $\left(e_{1}, e_{2}\right)=(0.80,1.60),(\mathrm{b})\left(e_{1}, e_{2}\right)=$ $(0.80,1.80),(\mathrm{c})\left(e_{1}, e_{2}\right)=(0.90,1.60)$ and $(\mathrm{d})\left(e_{1}, e_{2}\right)=(0.90,1.80)$ with $E_{k}=e_{k} \frac{\pi \rho_{0} c_{0}^{2}}{V_{\mathrm{m}}} \times$ $10^{-6}$ for $k=1$ and 2 . One solution (white zone), three solutions (black zone) and five solutions (grey zone).

For $\left(e_{1}, e_{2}\right)=(0.80,1.60)$, four small zones with three solutions appear (see Fig. 4 (a)) included in a large zone with one solution, stable only in a limited area (see Fig. 5 (a)). Two tongues in the $\sigma_{1} \omega_{1}^{-1}$-direction defining stable solutions are included into the instability zone showing that the system behavior is not similar considering the $\sigma_{1} \omega_{1}^{-1}$-direction and the $\sigma_{2} \omega_{2}^{-1}$ direction. Moreover, it is interesting to note that for $\left(e_{1}, e_{2}\right)=(0.80,0$. $)$ and $\left(e_{1}, e_{2}\right)=(0 ., 1.60)$ (see periodic case) unstable solutions were not observed. 

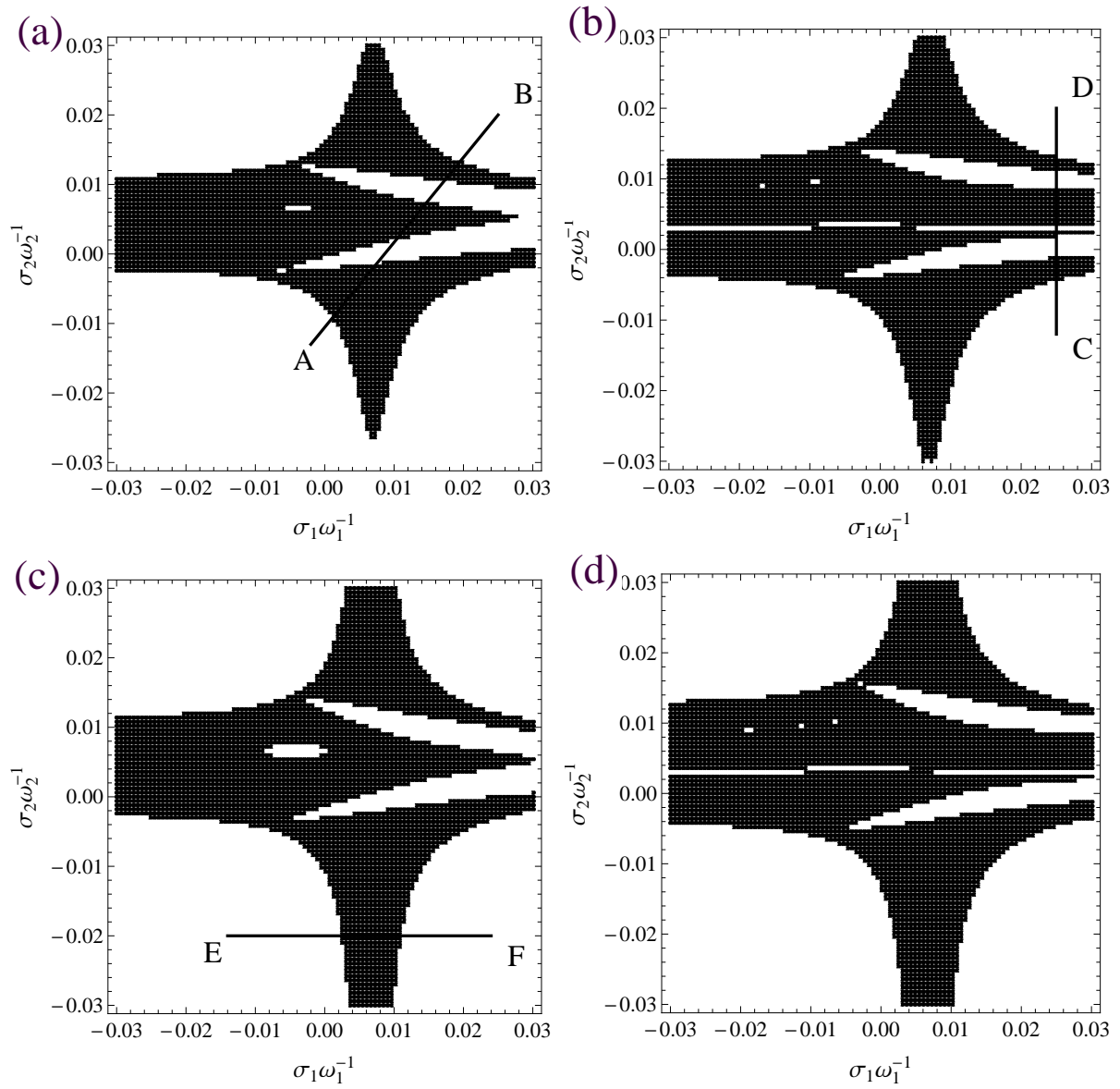

Figure 5: Stability zones of the fixed points of Eqs (58-63) for (a) $\left(e_{1}, e_{2}\right)=(0.80,1.60)$, (b) $\left(e_{1}, e_{2}\right)=(0.80,1.80),(\mathrm{c})\left(e_{1}, e_{2}\right)=(0.90,1.60)$ and $(\mathrm{d})\left(e_{1}, e_{2}\right)=(0.90,1.80)$ with $E_{k}=e_{k} \frac{\pi \rho_{0} c_{0}^{2}}{V_{\mathrm{m}}} \times 10^{-6}$ for $k=1$ and 2 . Zero stable solution (black zone) and one stable solution (white zone).

A first potential extension of the TET efficiency range appears: a detuned perturbation added to a main excitation could trigger the SMR mode when the amplitude threshold for self-triggering is not reached.

When increasing $e_{1}$ and/or $e_{2}$, the previous patterns (in terms of number of stationary solutions and stability zones) are reproduced and amplified along the axis $\sigma_{1} \omega_{1}^{-1}=0$ and/or $\sigma_{2} \omega_{2}^{-1}=0$ (see Figs 4 (b,c,d) and 5 (b,c,d)).

It is now interesting to check the validity of the complexification approach 
combined to the averaging method. This can be done by comparing as in the periodic case the multi-frequency-response diagrams in terms of $\max _{t \in\left[t_{1}, t_{2}\right]}\left|x_{i}(t)\right|$ for $i=1,2$ and 3 obtained from the fixed points of Eqs (58-63), by numerical integration of Eqs (58-63) using the fixed point as initial conditions $\left(t_{0}=0\right)$ and by numerical integration of the associated underlying linear system of Eqs. (37-39).

For $\left(e_{1}, e_{2}\right)=(0.80,1.60)$, the multi-frequency responses along the segment line $A B$ in the plane $\left(\sigma_{1} \omega_{1}^{-1}, \sigma_{2} \omega_{2}^{-1}\right)$ (see Figs. 4 and 5) are shown on Fig. 6. The segment line $A B$ is parametrized by $s$ where $s=0$ corresponds to the point $A$ and $s=1$ to the point $B$. For $s \approx 0.5$, the excitation frequencies of the associated bi-periodic excitation are near to the two resonance frequencies $\omega_{1}^{\mathrm{va}}$ and $\omega_{2}^{\mathrm{va}}$. The stable quasiperiodic solutions are well predicted by the analytical approach outside the instability zone (for $s \leq 0.1$ and $s \geq 0.96$ ) as well as on the stable tongue zone inside the instability zone. Finally in the zones correspondng to unstable fixed point solutions, the responses obtained by numerical integration differ from the responses obtained by the analytical approach. Using $s$ as the control parameter, a bifurcation analysis can be carried out. The results are reported on Fig. 6 for the $x_{3}$ component showing SN and Hopf bifurcation points.
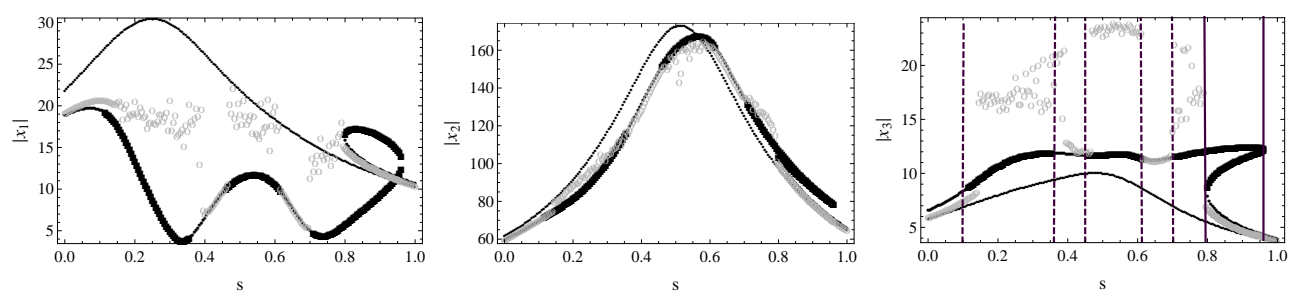

Figure 6: $\max _{t \in\left[t_{1}, t_{2}\right]}\left|x_{i}(t)\right|$ for $i=1,2$ and 3 obtained from the fixed points of Eqs (5863) (black curve with square markers), by numerical integration of Eqs. (37-39) (grey circle markers) and by numerical integration of the associated underlying linear system of Eqs. (37-39) (black continuous curve) versus $s$ the parametrization of the segment line $A B$ in the plane $\left(\sigma_{1} \omega_{1}^{-1}, \sigma_{2} \omega_{2}^{-1}\right)$ (see Figs. 4 and 5). Square markers denote unstable fixed point solutions and vertical dashed (respectively continuous) lines refer to Hopf (respectively SN) bifurcations. $e_{1}=0.80$ and $e_{2}=1.60\left(E_{k}=e_{k} \frac{\pi \rho_{0} c_{0}^{2}}{V_{\mathrm{m}}} \times 10^{-6}\right.$ for $k=1$ and 2$)$.

The same comments can be made on Fig. 7 (respectively Fig. 8) where the multi-frequency responses obtained with $\left(e_{1}, e_{2}\right)=(0.90,1.60)$ (respectively $\left.\left(e_{1}, e_{2}\right)=(0.80,1.80)\right)$ along the segment line $E F$ (respectively $\left.C D\right)$ in the 
plane $\left(\sigma_{1} \omega_{1}^{-1}, \sigma_{2} \omega_{2}^{-1}\right)$ (see Figs. 4 and 5) are shown. These two configuration can be compared respectively to the periodic cases: $\left(e_{1}, e_{2}\right)=(0.90,0$.) (see Fig. $3(\mathrm{a}-\mathrm{c}))$ and $\left(e_{1}, e_{2}\right)=(0 ., 1.80)$ (see Fig. $\left.3(\mathrm{~d}-\mathrm{f})\right)$. This comparison shows again that the system behavior is not similar considering the $\sigma_{1} \omega_{1}^{-1}$ direction and the $\sigma_{2} \omega_{2}^{-1}$-direction. The results along the segment line $E F$ are very close to the periodic case $\left(e_{1}, e_{2}\right)=(0.90,0$. $)$ where only the $x_{2}$ component is amplified. Conversely, the results along the segment line $C D$ differ significantly from the periodic case $\left(e_{1}, e_{2}\right)=(0 ., 1.80)$.

In all the situations exposed above, it can be noticed that $\max _{i}\left(\max _{t}\left|x_{i}(t)\right|\right)$ is always lower than the maximum value of the equivalent set of variables obtained from the underlying linear system (solid lines in Figs. 3, 6-8). In simple words, it means that the addition of a NES to this linear system reduces its maximal amplitude of vibration: a single NES has an effective action as a vibration limiter simultaneously on the two resonances of a linear system.
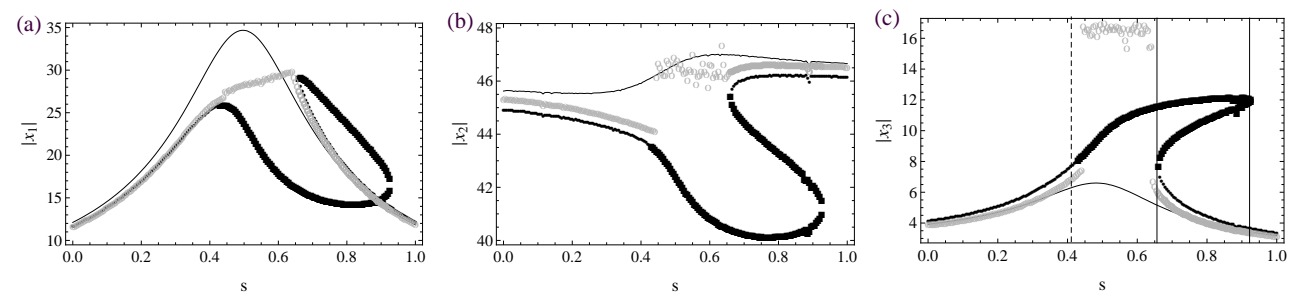

Figure 7: $\max _{t \in\left[t_{1}, t_{2}\right]}\left|x_{i}(t)\right|$ for $i=1,2$ and 3 obtained from the fixed points of Eqs (5863) (black curve with square markers), by numerical integration of Eqs. (37-39) (grey circle markers) and by numerical integration of the associated underlying linear system of Eqs. (37-39) (black continuous curve) versus $s$ the parametrization of the segment line $E F$ in the plane $\left(\sigma_{1} \omega_{1}^{-1}, \sigma_{2} \omega_{2}^{-1}\right)$ (see Figs. 4 and 5). Square markers denote unstable fixed point solutions and vertical dashed (respectively continuous) lines refer to Hopf (respectively SN) bifurcations. Square and circle markers denote unstable fixed point solutions. $e_{1}=0.90$ and $e_{2}=1.60\left(E_{k}=e_{k} \frac{\pi \rho_{0} c_{0}^{2}}{V_{\mathrm{m}}} \times 10^{-6}\right.$ for $k=1$ and 2$)$.

\subsection{Numerical verification}

This numerical verification has several objectives. First, it is a partial cross-check of the proposed analysis because it was carried out from the vibroacoustic model and with a different solver. The dimensional equations of motion Eqs. (10-12) were solved with (C)Matlab ordinary differential equation 

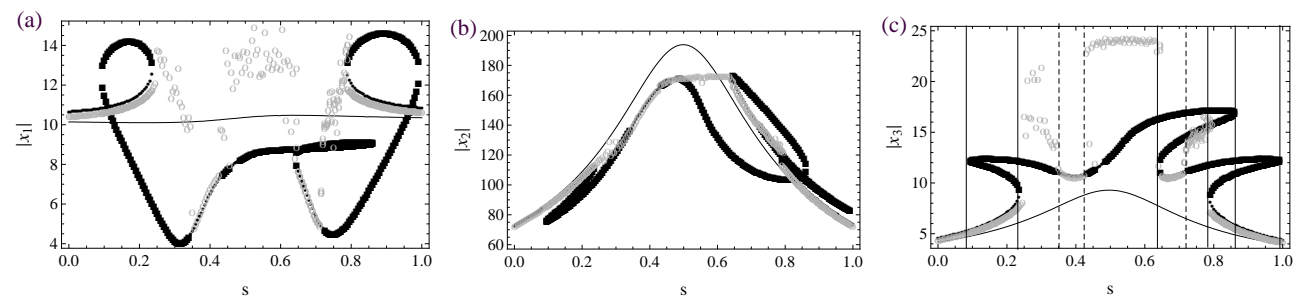

Figure 8: $\max _{t \in\left[t_{1}, t_{2}\right]}\left|x_{i}(t)\right|$ for $i=1,2$ and 3 obtained from the fixed points of Eqs (5863) (black curve with square markers), by numerical integration of Eqs. (37-39) (grey circle markers) and by numerical integration of the associated underlying linear system of Eqs. (37-39) (black continuous curve) versus $s$ the parametrization of the segment line $C D$ in the plane $\left(\sigma_{1} \omega_{1}^{-1}, \sigma_{2} \omega_{2}^{-1}\right)$ (see Figs. 4 and 5). Square markers denote unstable fixed point solutions and vertical dashed (respectively continuous) lines refer to Hopf (respectively SN) bifurcations. Square and circle markers denote unstable fixed point solutions. $e_{1}=0.80$ and $e_{2}=1.80\left(E_{k}=e_{k} \frac{\pi \rho_{0} c_{0}^{2}}{V \mathrm{~m}} \times 10^{-6}\right.$ for $k=1$ and 2$)$.

solver (Runge-Kutta $(4,5)$ formula), versus (C) Mathematica ordinary differential equations solver NDSolve (with the choice Automatic for the option Method) used with the nondimensional system (see previous Section). For the same parameters, the difference of the results obtained with the two solvers are in agreement with the precision of the numerical methods. Second, it permits to visualize the form and frequency content of the unstable responses. Third, it gives access to inner phenomena such as the spatial and temporal localization of the energy dissipation.

In the next subsections we give results obtained along the segment lines traced on Fig. 4. The dimensional equations of motion Eqs. (10-12) were solved assuming zero initial conditions $\left(t_{0}=0\right)$.

\subsubsection{Frequency analysis around the quasi-periodic regimes}

Fig. 9 displays the discrete Fourier transforms (Fast Fourier Transform (FFT) method) of the displacement of the membrane $q_{m}(t)$ for $t \in[0,30]$ (with frequency steps : $0.2 \mathrm{rad} / \mathrm{s}$ ) obtained for different values of $s(0.17$, 0.71 , and 0.79 ) on CD segment line in Fig. 8. In all the curves, the two main peaks correspond to the excitation frequencies. The other features of the curves differ.

Fig. 9 (a) corresponds to a quasi-periodic solution $(s=0.17$ in Fig. 8 (c)). Two peaks only are visible. 
Fig. 9 (b) corresponds to the vicinity of a Hopf bifurcation $(s=0.71$ in Fig. 8 (c)) according to the analysis performed in Section 3. We notice the presence of satellite peak around the main peaks. The distance between the main and satellite peaks is $22 \mathrm{rad} / \mathrm{s}$, a value close to the difference between the main peak and the imaginary part of the complex eigenvalues that characterize the Hopf bifurcation. There is no simple linear combination of the source frequencies that gives a result close to this value, and these frequencies here can be considered incommensurable. Thus, these peaks do not come from interaction of the excitation frequencies, so the simulated results are consistent with the analytic approach.

Fig. 9 (c) corresponds to the vicinity of a SN bifurcation $(s=0.79$ in Fig. 8 (c)) according to the analysis performed in Section 3. Here we do not notice clear satellite peaks, only a noisy background is present as a trace of non harmonic features. There is no indication of an imaginary part in the eigenvalues that arise at this bifurcation point, which is consistent with a SN bifurcation.
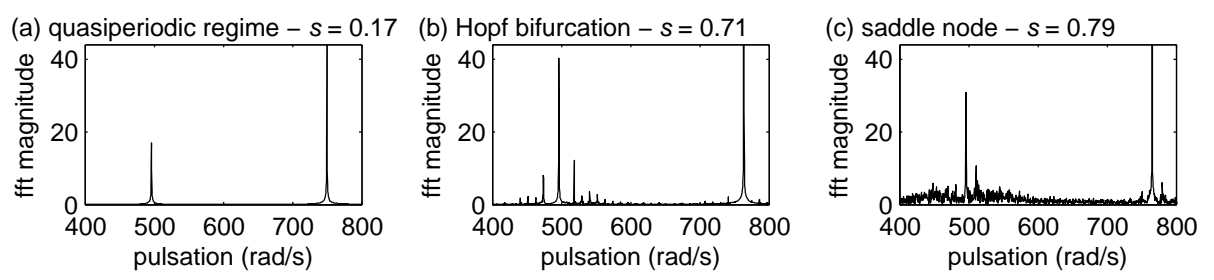

Figure 9: Fast Fourier Transform of $q_{m}(t)$ with $t$ in [0 $\left.30 \mathrm{~s}\right]$ for different values of $s$ on the segment line CD. (a) $s=0.17\left(\omega_{1}=496 \mathrm{rad} / \mathrm{s}, \omega_{2}=750 \mathrm{rad} / \mathrm{s}\right)$, (b) $s=0.71$ $\left(\omega_{1}=496 \mathrm{rad} / \mathrm{s}, \omega_{2}=764 \mathrm{rad} / \mathrm{s}\right),(\mathrm{c}) s=0.79\left(\omega_{1}=496 \mathrm{rad} / \mathrm{s}, \omega_{2}=765 \mathrm{rad} / \mathrm{s}\right)$.

\subsubsection{Dissipated power in $S M R$}

For a system with one tube only, as studied before [7, 8], a harmonic excitation can produce a strongly modulated response, indicating an alternation of modes corresponding successively to resonance build up and TET. For more complicated systems, the analysis is less easy but the same ideas can be considered as a basis of reflection.

We have found temporal responses in the central unstable part of the segments CD (see Fig. 8) and EF (see Fig. 7), where a strongly modulated pattern is observed. To analysis the pattern, the powers dissipated in the three subsystems (the pipe 1, the pipe 2 and the membrane) have been 
compared. The power dissipated in a subsystem was obtained by making the product of the opposite of the dissipative forces with the speed of the corresponding variables characterizing the subsystem. With the notations used in Eqs. (10-12), we get

$$
\begin{aligned}
P_{1}(t) & =2 \tau_{1} \sqrt{k_{1} m_{1}} \dot{u}_{1}^{2}(t) \\
P_{2}(t) & =2 \tau_{2} \sqrt{k_{2} m_{2}} \dot{u}_{2}^{2}(t) \\
P_{m}(t) & =2 k_{3} \eta\left|q_{\mathrm{m}}(t)\right|^{2} \dot{q}_{\mathrm{m}}^{2}(t)
\end{aligned}
$$

where $P_{i}$ denotes the power dissipated in the tube $i$, and $P_{m}$ denotes the power dissipated in the membrane.

We chose this representation for two reasons. First, we want to distinguish TET from resonance build-up: we expect a strong dissipation in the NES during TET. Second, this representation does not differ fundamentally from the velocity or displacement representations: if the systems were not coupled, the amplitude ratio between the velocity and displacement would be a constant, and the dissipation amplitude would have an amplitude proportional to the square of these quantities. Note that we also used the flux of mechanical energy between the different components of the system in order to check the conservation of energy (not shown here).

Fig. 10 (a) corresponds to the point $s=0.53$ in the segment line CD (see Fig. $8(\mathrm{c}))$. Here the source is tuned at the resonance peak of the highest main linear mode. It is close to the resonance peak of tube 2 although it is higher because of the stiffness added by the coupling box. The source is detuned for the lowest main linear mode (which is close to the resonance peak of tube 1).

We observe almost no dissipation in tube 1 (top curve), and some dissipation occurs in tube 2 and in the membrane (the NES). The responses display a strong modulation : the magnitude of the amplitude modulation of the response is equal to its amplitude, like the SMR reported in $[12,5]$ ). More precisely, an alternation of two regimes can be observed. One regime corresponds to resonance build-up in tube 2 (middle curve), for instance around the $19^{\text {th }}$ second: the source feeds the tube, with an amplitude too small to be balanced by the dissipation there, thus the amplitude (and dissipation) grows. There is clearly very few connection between the tube and the membrane since the latter one is not much excited (bottom curve). The second regime of the alternation corresponds to irreversible energy transfer from tube 2 to the membrane. There is a sudden burst in the membrane 

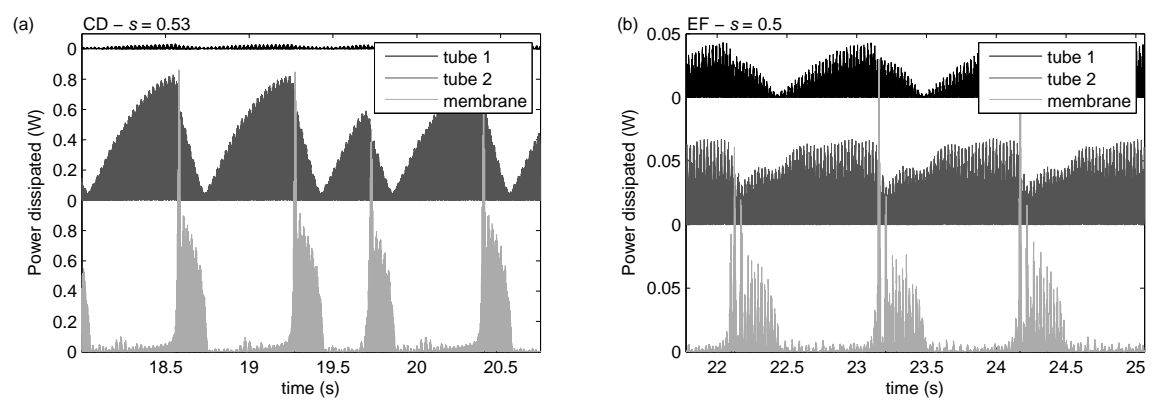

Figure 10: Power dissipated in the system as a function of time. From top to bottom: power dissipated in tube 1 , tube 2 , and in the membrane. (a) $s=0.53$ in $\mathrm{CD}\left(\omega_{1}=496 \mathrm{rad} / \mathrm{s}, \omega_{2}=759 \mathrm{rad} / \mathrm{s}\right),(\mathrm{b}) s=0.5$ in $\mathrm{EF}\left(\omega_{1}=485 \mathrm{rad} / \mathrm{s}\right.$, $\left.\omega_{2}=740 \mathrm{rad} / \mathrm{s}\right)$. The time scale is large compared to the excitation periods $(8 \mathrm{~ms}$ and $13 \mathrm{~ms}$ ). The light grey bottom curves peak at values close to $1.5 \mathrm{~W}$ (a) and $0.24 \mathrm{~W}$ (b) and overlap the other curves. The teeth in the curves correspond to a 25 ms modulation for both graphs.

dissipation (roughly two orders of magnitude) and a decrease in the tube 2 dissipation indicating a decrease in amplitude there despite the source activity. This is a clear similarity with simpler systems, although a closer look shows a modulation in dissipation in the two regimes.

Fig. 10 (b) corresponds to the point $s=0.50$ in the segment line EF (see Fig. 7 (c)). Here the source is tuned at the resonance peak of the lowest main linear mode (close to the resonance of tube 1). The source is detuned for the highest main linear mode (close to the resonance of tube 2 ).

Here the observations differ slightly from the upper ones. The membrane and tube 1 behave in accordance with the alternation regime described above, but dissipation is important in tube 2 . It seems that tube 2 amplitude reaches a limit, too small to trigger TET. It seems also that a part of the energy in tube 2 is quickly flushed away when the tube 1 triggers TET. The main goal of this section being a check of the analytical results of Section 3, we did not analyze these observations deeper. In particular we neglected here the various couplings in the system.

\section{Conclusions}

In the framework of NES properties exploration, we studied a 2 DOF linear system weakly coupled to a NES and submitted to a quasi-periodic 
excitation near its main resonance frequencies. We used different methods in order to describe its behavior, among them complexification and numerical integration of motion equations, and we analyzed the results in terms of stability, frequency content, and energy dissipation.

We observed different regimes ascribed to periodic, quasi-periodic or SMR regimes, and we cross-checked the consistency of the methods when possible.

We proposed a method to approach the quasi-periodic solutions as roots of a polynomial and to determine their stability. We observed that in theory, a detuned perturbation can trigger TET, that TET in one part of a system can flush away energy in another part, and that a single NES can limit the greatest vibration amplitude although the system is excited simultaneously around its two main resonance frequencies with comparable amplitudes.

This theoretical and numerical work paves the way for future experiments. It would also be interesting to analyze more thoroughly the unstable solutions, in the perspective of broadening the applications of NES for complex and permanent excitations.

\section{References}

[1] B. Cochelin, P. Herzog, P.-O. Mattei, Experimental evidence of energy pumping in acoustics, C. R. Mécanique 334 (11) (2006) 639-644.

[2] R. Bellet, B. Cochelin, P. Herzog, P.-O. Mattei, Experimental study of targeted energy transfer from an acoustic system to a nonlinear membrane absorber, Journal of Sound and Vibration 329 (2010) 2768-2791.

[3] R. Mariani, S. Bellizzi, B. Cochelin, P. Herzog, P.-O. Mattei, Toward an adjustable nonlinear low frequency acoustic absorber, Journal of Sound and Vibration 330 (2011) 5245-5258.

[4] R. Bellet, B. Cochelin, R. Côte, P.-O. Mattei, Enhancing the dynamic range of targeted energy transfer in acoustics using several nonlinear membrane absorbers, Journal of Sound and Vibration 331 (26) (2012) $5657-5668$.

[5] A. Vakakis, O. Gendelman, L. Bergman, D. McFarland, G. Kerschen, Y. Lee, Nonlinear targeted energy transfer in mechanical and structural systems, Vol. 156 of Solid mechanics and its applications, Springer, 2008. 
[6] E. Gourdon, N. Alexander, C. Taylor, C. Lamarque, S. Pernot, Nonlinear energy pumping under transient forcing with strongly nonlinear coupling: Theoretical and experimental results, Journal of Sound and Vibration 300 (2007) 522-551.

[7] Y. Starosvetsky, O. Gendelman, Response regimes of linear oscillator coupled to nonlinear energy sink with harmonic forcing and frequency detuning, Journal of Sound and Vibration 315 (2008) 746-765.

[8] O. V. Gendelman, Y. Starosvetsky, M. Feldman, Attractors of harmonically forced linear oscillator with attached nonlinear energy sink i: Description of response regimes, Nonlinear Dynamics 51 (2008) 31-46.

[9] Y. Starosvetsky, O. V. Gendelman, Attractors of harmonically forced linear oscillator with attached nonlinear energy sink. ii: Optimization of a nonlinear vibration absorber, Nonlinear Dynamics 51 (2008) 47-57.

[10] Y. Starosvetsky, O. Gendelman, Vibration absorption in systems with a nonlinear energy sink: Nonlinear damping, Journal of Sound and Vibration 324 (2009) 916-939.

[11] T. Pham, S. Pernot, C. Lamarque, Competitive energy transfer between a two degree-of-freedom dynamic system and an absorber with essential nonlinearity, Nonlinear Dynamics 62 (2010) 573-592.

[12] Y. Starosvetsky, O. Gendelman, Dynamics of a strongly nonlinear vibration absorber coupled to a harmonically excited two-degree-of-freedom system, Journal of Sound and Vibration 312 (2008) 234-256.

[13] Y. Lee, A. Vakakis, L. Bergman, D. McFarland, G. Kerschen, Suppression of aeroelastic instabilities by means of targeted energy transfers: Part I, theory, AIAA Journal 45 (3) (2007) 693-711.

[14] Y. Kim, S. Choi, A multiple harmonic balance method for the internal resonant vibration of a nonlinear jeffcott rotro, Journal of Sound and Vibration 208 (1997) 745-761.

[15] M. Guskov, J. Sinou, F. Thouverez, Multi-dimensional harmonic balance applied to rotor dynamics, Mechanics Research Communications 35 (2008) 537-545. 


\section{Table captions}

- Table 1: Resonance frequencies. 


\section{Figure captions}

- Figure 1: Schema of the vibroacoustic system.

- Figure 2: (a-c): $\left|\varphi_{i}^{10}\right|$ for $i=1,2$ and 3 with $e_{1}=0.80$ (black) and 0.90 (grey) and $e_{2}=0$. (d-f): $\left|\varphi_{i}^{20}\right|$ for $i=1,2$ and 3 with and $e_{1}=0$ and $e_{2}=1.60$ (black) and 1.80 (grey). Stable solutions (dot markers), unstable solutions (circle markers). $\quad\left(E_{k}=e_{k} \frac{\pi \rho_{0} c_{0}^{2}}{V_{\mathrm{m}}} \times 10^{-6}\right.$ for $k=$ 1 and 2).

- Figure 3: $\max _{t \in\left[t_{1}, t_{2}\right]}\left|x_{i}(t)\right|$ for $i=1,2$ and 3 obtained from the fixed points of Eqs (58-63) (black), by numerical integration of Eqs. (37-39) (circle markers) and by numerical integration of the associated underlying linear system of Eqs. (37-39) (continuous curves). (a-c): $e_{1}=0.90$ and $e_{2}=0$. (d-f): $e_{1}=0$ and $e_{2}=1.80$. Filled square markers denote unstable fixed point solutions. $\left(E_{k}=e_{k} \frac{\pi \rho_{0} c_{0}^{2}}{V_{\mathrm{m}}} \times 10^{-6}\right.$ for $k=1$ and 2$)$.

- Figure 4: Number of fixed points of Eqs (58-63) for (a) $\left(e_{1}, e_{2}\right)=$ $(0.80,1.60),(\mathrm{b})\left(e_{1}, e_{2}\right)=(0.80,1.80),(\mathrm{c})\left(e_{1}, e_{2}\right)=(0.90,1.60)$ and $(\mathrm{d})$ $\left(e_{1}, e_{2}\right)=(0.90,1.80)$ with $E_{k}=e_{k} \frac{\pi \rho_{0} c_{0}^{2}}{V_{\mathrm{m}}} \times 10^{-6}$ for $k=1$ and 2 . One solution (white zone), three solutions (black zone) and five solutions (grey zone).

- Figure 5: Stability zones of the fixed points of Eqs (58-63) for (a) $\left(e_{1}, e_{2}\right)=(0.80,1.60),(\mathrm{b})\left(e_{1}, e_{2}\right)=(0.80,1.80),(\mathrm{c})\left(e_{1}, e_{2}\right)=(0.90,1.60)$ and $(\mathrm{d})\left(e_{1}, e_{2}\right)=(0.90,1.80)$ with $E_{k}=e_{k} \frac{\pi \rho_{0} c_{0}^{2}}{V_{\mathrm{m}}} \times 10^{-6}$ for $k=1$ and 2 . Zero stable solution (black zone) and one stable solution (white zone).

- Figure 6: $\max _{t \in\left[t_{1}, t_{2}\right]}\left|x_{i}(t)\right|$ for $i=1,2$ and 3 obtained from the fixed points of Eqs (58-63) (black curve with square markers), by numerical integration of Eqs. (37-39) (grey circle markers) and by numerical integration of the associated underlying linear system of Eqs. (37-39) (black continuous curve) versus $s$ the parametrization of the segment line $A B$ in the plane $\left(\sigma_{1} \omega_{1}^{-1}, \sigma_{2} \omega_{2}^{-1}\right)$ (see Figs. 4 and 5$)$. Square markers denote unstable fixed point solutions and vertical dashed (respectively continuous) lines refer to Hopf (respectively SN) bifurcations. $e_{1}=0.80$ and $e_{2}=1.60\left(E_{k}=e_{k} \frac{\pi \rho_{0} c_{0}^{2}}{V \mathrm{~m}} \times 10^{-6}\right.$ for $k=1$ and 2$)$. 
- Figure 7: $\max _{t \in\left[t_{1}, t_{2}\right]}\left|x_{i}(t)\right|$ for $i=1,2$ and 3 obtained from the fixed points of Eqs (58-63) (black curve with square markers), by numerical integration of Eqs. (37-39) (grey circle markers) and by numerical integration of the associated underlying linear sysetm of Eqs. (37-39) (black continuous curve) versus $s$ the parametrization of the segment line $E F$ in the plane $\left(\sigma_{1} \omega_{1}^{-1}, \sigma_{2} \omega_{2}^{-1}\right)$ (see Figs. 4 and 5$)$. Square markers denote unstable fixed point solutions and vertical dashed (respectively continuous) lines refer to Hopf (respectively SN) bifurcations. Square and circle markers denote unstable fixed point solutions. $e_{1}=0.90$ and $e_{2}=1.60\left(E_{k}=e_{k} \frac{\pi \rho_{0} c_{0}^{2}}{V \mathrm{~m}} \times 10^{-6}\right.$ for $k=1$ and 2$)$.

- Figure 8: $\max _{t \in\left[t_{1}, t_{2}\right]}\left|x_{i}(t)\right|$ for $i=1,2$ and 3 obtained from the fixed points of Eqs (58-63) (black curve with square markers), by numerical integration of Eqs. (37-39) (grey circle markers) and by numerical integration of the associated underlying linear system of Eqs. (37-39) (black continuous curve) versus $s$ the parametrization of the segment line $C D$ in the plane $\left(\sigma_{1} \omega_{1}^{-1}, \sigma_{2} \omega_{2}^{-1}\right)$ (see Figs. 4 and 5). Square markers denote unstable fixed point solutions and vertical dashed (respectively continuous) lines refer to Hopf (respectively SN) bifurcations. Square and circle markers denote unstable fixed point solutions. $e_{1}=0.80$ and $e_{2}=1.80\left(E_{k}=e_{k} \frac{\pi \rho_{0} c_{0}^{2}}{V_{\mathrm{m}}} \times 10^{-6}\right.$ for $k=1$ and 2$)$.

- Figure 9: Fast Fourier Transform of $q_{m}(t)$ with $t$ in $[030 \mathrm{~s}]$ for different values of $s$ on the segment line CD. (a) $s=0.17\left(\omega_{1}=496 \mathrm{rad} / \mathrm{s}\right.$, $\left.\omega_{2}=750 \mathrm{rad} / \mathrm{s}\right),(\mathrm{b}) s=0.71\left(\omega_{1}=496 \mathrm{rad} / \mathrm{s}, \omega_{2}=764 \mathrm{rad} / \mathrm{s}\right)$, (c) $s=0.79\left(\omega_{1}=496 \mathrm{rad} / \mathrm{s}, \omega_{2}=765 \mathrm{rad} / \mathrm{s}\right)$.

- Figure 10: Power dissipated in the system as a function of time. From top to bottom: power dissipated in tube 1, tube 2, and in the membrane. (a) $s=0.53$ in $\mathrm{CD}\left(\omega_{1}=496 \mathrm{rad} / \mathrm{s}, \omega_{2}=759 \mathrm{rad} / \mathrm{s}\right)$, (b) $s=0.5 \mathrm{in} \mathrm{EF}\left(\omega_{1}=485 \mathrm{rad} / \mathrm{s}, \omega_{2}=740 \mathrm{rad} / \mathrm{s}\right)$. The time scale is large compared to the excitation periods $(8 \mathrm{~ms}$ and $13 \mathrm{~ms})$. The light grey bottom curves peak at values close to $1.5 \mathrm{~W}$ (a) and $0.24 \mathrm{~W}$ (b) and overlap the other curves. The teeth in the curves correspond to a $25 \mathrm{~ms}$ modulation for both graphs. 